\title{
MAXIMAL FUNCTION CHARACTERIZATIONS FOR HARDY SPACES ON SPACES OF HOMOGENEOUS TYPE WITH FINITE MEASURE AND APPLICATIONS
}

\author{
THE ANH BUI, XUAN THINH DUONG, AND FU KEN LY
}

\begin{abstract}
We prove nontangential and radial maximal function characterizations for Hardy spaces associated to a non-negative self-adjoint operator satisfying Gaussian estimates on a space of homogeneous type with finite measure. This not only addresses an open point in the literature, but also gives a complete answer to the question posed by Coifman and Weiss in the case of finite measure. We then apply our results to give maximal function characterizations for Hardy spaces associated to second order elliptic operators with Neumann and Dirichlet boundary conditions, Schrödinger operators with Dirichlet boundary conditions, and FourierBessel operators.
\end{abstract}

\section{Contents}

1. Introduction

2. Some kernel and maximal function estimates

3. Maximal function characterizations for Hardy spaces $H_{L}^{p}(X)$

3.1. Proof of Theorem 1.4

3.2. Proof of Theorem 1.8

4. Applications 14

4.1. Second-order elliptic operators with Neumann boundary conditions 14

4.2. Second-order elliptic operators with Dirichlet boundary conditions $\quad 15$

4.3. Schrödinger operators with Dirichlet boundary conditions $\quad 19$

4.4. Fourier-Bessel operators on $((0,1), d x) \quad 22$

4.5. Fourier-Bessel operators on $\left((0,1), x^{2 \nu+1} d x\right) \quad 28$

References $\quad 35$

\section{Introduction}

Let $(X, d, \mu)$ be a metric space endowed with a nonnegative Borel measure $\mu$ satisfying the following 'doubling' condition: there exists a constant $C_{1}>0$ such that

$$
\mu(B(x, 2 r)) \leq C_{1} \mu(B(x, r))
$$

for all $x \in X, r>0$ and all balls $B(x, r):=\{y \in X: d(x, y)<r\}$. For the moment $\mu(X)$ may be finite or infinite.

It is not difficult to see that the condition (1) implies that there exists a "dimensional" constant $n \geq 0$ so that

$$
\mu(B(x, \lambda r)) \leq C_{2} \lambda^{n} \mu(B(x, r))
$$

for all $x \in X, r>0$ and $\lambda \geq 1$, and

$$
\mu(B(x, r)) \leq C_{3} \mu(B(y, r))\left(1+\frac{d(x, y)}{r}\right)^{n}
$$

\footnotetext{
2010 Mathematics Subject Classification. 42B30, 42B35, 35K08, 35J25.

Key words and phrases. Hardy space, heat kernel, maximal function characterization, second order elliptic operator, Fourier-Bessel operator.
} 
for all $x, y \in X, r>0$.

Assume also the existence of an operator $L$ that satisfies the following two conditions:

(A1) $L$ is a nonnegative self-adjoint operator on $L^{2}(X)$;

(A2) $L$ generates a semigroup $\left\{e^{-t L}\right\}_{t>0}$ whose kernel $p_{t}(x, y)$ admits a Gaussian upper bound. That is, there exist two positive constants $C$ and $c$ so that for all $x, y \in X$ and $t>0$,

$$
\left|p_{t}(x, y)\right| \leq \frac{C}{\mu(B(x, \sqrt{t}))} \exp \left(-\frac{d(x, y)^{2}}{c t}\right) .
$$

Then for $0<p \leq 1$ one can define three notions of Hardy spaces related to $L$. The first notion is through linear combinations of atoms that appropriately encode the cancellation inherent in $L$. The second and third notions are $H_{L, \max }^{p}$ and $H_{L, \text { rad }}^{p}$, which are defined via the non-tangential maximal function and the radial maximal function respectively. For the reader's convenience we recall these notions below.

Definition 1.1 (Atoms for $L)$. Let $p \in(0,1]$ and $M \in \mathbb{N}$. A function a supported in a ball $B$ is called $a(p, M)_{L}$-atom if there exists a function $b \in \mathcal{D}\left(L^{M}\right)$ such that

(i) $a=L^{M} b$;

(ii) $\operatorname{supp} L^{k} b \subset B, k=0,1, \ldots, M$;

(iii) $\left\|L^{k} b\right\|_{L^{\infty}(X)} \leq r_{B}^{2(M-k)} \mu(B)^{-\frac{1}{p}}, k=0,1, \ldots, M$.

In the particular case where $\mu(X)<\infty$, the constant function $[\mu(X)]^{-1 / p}$ is also considered as an atom.

Then the atomic Hardy space associated to the operator $L$ are defined as follows:

Definition 1.2 (Atomic Hardy spaces for $L$ ). Given $p \in(0,1]$ and $M \in \mathbb{N}$, we say that $f=$ $\sum \lambda_{j} a_{j}$ is an atomic $(p, M)_{L}$-representation if $\left\{\lambda_{j}\right\}_{j=0}^{\infty} \in l^{p}$, each $a_{j}$ is a $(p, M)_{L}$-atom, and the sum converges in $L^{2}(X)$. The space $H_{L, a t, M}^{p}(X)$ is then defined as the completion of

$$
\left\{f \in L^{2}(X): f \text { has an atomic }(p, M)_{L} \text {-representation }\right\} \text {, }
$$

with the norm given by

$$
\|f\|_{H_{L, a t, M}^{p}(X)}=\inf \left\{\left(\sum\left|\lambda_{j}\right|^{p}\right)^{1 / p}: f=\sum \lambda_{j} a_{j} \text { is an atomic }(p, M)_{L} \text {-representation }\right\} .
$$

The maximal Hardy spaces associated to $L$ are defined as follows.

Definition 1.3 (Maximal Hardy spaces for $L$ ). For $f \in L^{2}(X)$, we define the non-tangential maximal function associated to $L$ of $f$ by

$$
f_{L}^{*}(x)=\sup _{t>0} \sup _{d(x, y)<t}\left|e^{-t L} f(y)\right|
$$

and the radial maximal function by

$$
f_{L}^{+}(x)=\sup _{t>0}\left|e^{-t L} f(x)\right| .
$$

Given $p \in(0,1]$, the Hardy space $H_{L, \max }^{p}(X)$ is defined as the completion of

$$
\left\{f \in L^{2}(X): f_{L}^{*} \in L^{p}(X)\right\}
$$

with the norm given by

$$
\|f\|_{H_{L, \max }^{p}(X)}=\left\|f_{L}^{*}\right\|_{L^{p}(X)} .
$$

Similarly, the Hardy space $H_{L, \mathrm{rad}}^{p}(X)$ is defined as the completion of

$$
\left\{f \in L^{2}(X): f_{L}^{+} \in L^{p}(X)\right\}
$$

with the norm given by

$$
\|f\|_{H_{L, \mathrm{rad}}^{p}(X)}=\left\|f_{L}^{+}\right\|_{L^{p}(X)} .
$$


The theory of Hardy spaces associated to differential operators was initiated in [1] and since then the theory has been studied intensively by many mathematicians. See for example $[17$, $2,23,22,24]$ and the references therein. In this framework it is understood that the classical Hardy spaces $H^{p}\left(\mathbb{R}^{n}\right)$ can be viewed as the Hardy spaces associated to the Laplacian $-\Delta$.

A substantive problem in the theory of Hardy spaces is to determine conditions for which the atomic and maximal notions coincide, and it this problem which is the focus of our paper. More precisely we wish to answer the following question:

Question: Does the following equivalence hold:

$$
H_{L, a t, M}^{p}(X) \equiv H_{L, \max }^{p}(X) \equiv H_{L, \mathrm{rad}}^{p}(X)
$$

for sufficiently large $M$ ?

Before presenting our main result we highlight some history and known results related to (4).

(i) In the Euclidean setting, when $L=-\Delta$, the Hardy spaces associated to $L$ and the classical Hardy spaces are identical. The classical Hardy spaces has its roots in complex function theory, and it was in that setting that the connection with the non-tangential maximal function was first elucidated [5]. The role of maximal functions then took centre stage and was instrumental in the development of the real-variable theory beginning with the seminal work of Fefferman and Stein [21]. From that point onwards the theory developed rapidly and, through the efforts of $[11,25,26,7,8]$, the atomic characterization was added to the fold.

(ii) The notion of atoms enabled the extension of Hardy spaces from $\mathbb{R}^{n}$ to other structures [12], and it was there that Coifman and Weiss introduced the concept of a space of homogeneous type. The viewpoint, as espoused in [12], was to develop the theory on $X$ by starting with the notion of atomic Hardy spaces, which we shall denote by $H_{C W}^{p}(X)$ for $\frac{n}{n+1}<p \leq 1$ (see Definition 1.7 below). Under certain additional geometric assumptions, Coifman and Weiss proved the radial maximal function characterization for $H_{C W}^{1}(X)$. They then proposed the following question:

Question (Coifman-Weiss): Can one characterize the Hardy spaces $H_{C W}^{p}(X)$ by maximal functions for $p$ below 1 ?

This question has been partly answered in the setting of Ahlfors $n$-regular metric measure spaces. Recall that such spaces are spaces of homogeneous type with $\mu(B(x, r)) \sim r^{n}$ for all $x \in X$ and $r \in(0,2 \operatorname{diam}(X))$. When $X$ is an Ahlfors 1-regular metric measure space Uchiyama [34] proved that the spaces $H_{C W}^{p}(X)$ can be characterized by radial maximal functions for $p<1$, but unfortunately the range of $p$ in [34] is not optimal. The same result was obtained by [27] for the range $1 / 2<p \leq 1$. A complete answer was given by [35] but extra structural assumptions are needed - namely a so called reverse-doubling condition on $\mu$ and that $\mu(X)=\infty$. To the best of our knowledge, the remaining case $\mu(X)<\infty$ is non-trivial and is still open.

(iii) In our setting, the theory of Hardy spaces arises from the fundamental observation that the classical Hardy spaces on $\mathbb{R}^{n}$ is intrinsically tied to the Laplacian $-\Delta$ and this observation allows the theory to be generalized in another direction. The articles [23, 22] give an account of this body of work and there one can also find partial answers to (4). The full equivalence was proved $[14,32,33]$ but further assumptions were required in addition to (A1) and (A2). Reverse-doubling on $X$ and a regularity and markov condition on $L$ (see (A3) and (A4) below) was required in [14], while $\mu(X)=\infty$ was implicitly required in $[32,33]$. It is worth mentioning that the proofs in [32, 33], which are an adaptation of [7], does not work well in the case $\mu(X)<\infty$ and thus, in this situation, the problem is still open.

This brings us to the first goal of the present article, which is to address the finite case in (iii) above. More precisely we shall prove 
Theorem 1.4. Let $\mu(X)<\infty$ and assume $L$ satisfies (A1) and (A2). Let $p \in(0,1]$, and $M>\frac{n}{2}\left(\frac{1}{p}-1\right)$. Then the Hardy spaces $H_{L, a t, M}^{p}(X), H_{L, \max }^{p}(X)$ and $H_{L, \mathrm{rad}}^{p}(X)$ coincide with equivalent norms.

Let us explain the relevance of the condition $\mu(X)=\infty$ in $[32,33]$. The proofs there are rooted in decomposition of the product space $X \times(0, \infty)$, which we sketch here for the sake of convenience. For each $i \in \mathbb{Z}$ one defines the level set $O_{i}:=\left\{x \in X: \mathbf{M} f(x)>2^{i}\right\}$ where $\mathbf{M}$ is a certain maximal function that is lower-continuous, and the tent of $O_{i}$ through $\widehat{O}_{i}:=(x, t) \in$ $X \times(0, \infty): B(x, 4 t) \subset O$. Then the space $X \times(0, \infty)$ can be decomposed as follows:

$$
X \times(0, \infty)=\bigcup_{i} \widehat{O}_{i} \backslash \widehat{O}_{i+1} .
$$

Unfortunately (5) fails in the case $X$ is bounded and this is the reason why the argument used in the case $\mu(X)=\infty$ is not applicable to the case $\mu(X)<\infty$. To overcome this obstacle, some new ideas are employed such as a new decomposition of $X \times(0, \infty)$. It is worth pointing out firstly that our approach is also applicable for the case $\mu(X)=\infty$ and secondly, that although our decomposition of the underlying product space $X \times(0, \infty)$ bears a resemblance to that in [14], the absence of both reverse-doubling on $X$ and the conditions (A3) and (A4) on $L$ requires some significant innovations and improvements. The details can be found in Section 3.

By combining Theorem 1.4 with Theorem 1.2 of [32] we can now state the following, completing the picture in point (iii) above.

Corollary 1.5. Let $\mu(X)$ be finite or infinite and assume $L$ satisfies (A1) and (A2). Let $p \in(0,1]$, and $M>\frac{n}{2}\left(\frac{1}{p}-1\right)$. Then the Hardy spaces $H_{L, a t, M}^{p}(X), H_{L, \max }^{p}(X)$ and $H_{L, \mathrm{rad}}^{p}(X) c o-$ incide with equivalent norms. Due to this coincidence, we shall write $H_{L}^{p}(X)$ for any $H_{L, a t, M}^{p}(X)$, $H_{L, \text { max }}^{p}(X)$ and $H_{L, \text { rad }}^{p}(X)$ for any such $p$ and $M$.

Our second goal is to give the answer for the question in (ii) proposed by Coifman and Weiss under the presence of an operator $L$ when $\mu(X)$ is finite. We first recall the definition of the Hardy spaces $H_{C W}^{p}(X)$ on $X$.

Definition 1.6 (p-atoms). Let $p \in\left(\frac{n}{n+1}, 1\right]$. A function a is called a p-atom associated to the ball $B$ if

(i) $\operatorname{supp} a \subset B$

(ii) $\|a\|_{L^{\infty}(X)} \leq \mu(B)^{-1 / p}$

(iii) $\int a(x) d \mu(x)=0$

When $\mu(X)<\infty$ then the constant function $\mu(X)^{-1 / p}$ is also an atom.

To define the Hardy space $H_{C W}^{p}$ for $p$ below 1 , we need to introduce the Lipschitz spaces $\mathfrak{L}_{\alpha}$. We say that the function $f$ is a member of $\mathfrak{L}_{\alpha}$ if there exists a constant $c>0$, such that

$$
|f(x)-f(y)| \leq c|B|^{\alpha}
$$

for all ball $B$ and $x, y \in B$, and the best constant $c$ can be taken to be the norm of $f$ and is denoted by $\|f\|_{\mathfrak{L}_{\alpha}}$.

Definition 1.7 (Hardy spaces of Coifman and Weiss). Let $\frac{n}{n+1}<p \leq 1$. We say that a function $f \in H_{C W}^{p}(X)$ if $f \in L^{1}(X)$ for $p=1$, or $f \in \mathfrak{L}_{1 / p-1}^{*}$ for $p<1$, and there exists a sequence $\left(\lambda_{j}\right)_{j \in \mathbb{N}} \in \ell^{p}$ and a sequence of $p$-atoms $\left(a_{j}\right)_{j \in \mathbb{N}}$ such that $f=\sum_{j} \lambda_{j} a_{j}$ in $L^{1}(X)$ for $p=1$, and $f=\sum_{j} \lambda_{j} a_{j}$ in $\mathfrak{L}_{1 / p-1}^{*}$ for $p<1$. We set

$$
\|f\|_{H_{C W}^{p}}=\inf \left\{\left(\sum_{j}\left|\lambda_{j}\right|^{p}\right)^{1 / p}: f=\sum_{j} \lambda_{j} a_{j}\right\} .
$$

We now consider the following two additional conditions for the operator $L$ : 
(A3) There exists $\delta \in(0,1]$ such that for every $d\left(y, y^{\prime}\right)<\sqrt{t} / 2$ and $0<t<\operatorname{diam} X$,

$$
\left|p_{t}(x, y)-p_{t}\left(x, y^{\prime}\right)\right| \leq\left(\frac{d\left(y, y^{\prime}\right)}{\sqrt{t}}\right)^{\delta} \frac{C}{\mu(B(x, \sqrt{t}))} \exp \left(-\frac{d(x, y)^{2}}{c t}\right) .
$$

(A4) For every $x \in X$ and $t>0$, we have

$$
\int_{X} p_{t}(x, y) d \mu(y)=1
$$

Then we have the following.

Theorem 1.8. Let $\mu(X)<\infty$ and assume the operator L satisfies (A1), (A2), (A3) and (A4). Let $p \in\left(\frac{n}{n+\delta}, 1\right]$ and $M>\frac{n}{2}\left(\frac{1}{p}-1\right)$. Then $H_{C W}^{p}(X), H_{L, \max }^{p}(X)$ and $H_{L, \mathrm{rad}}^{p}(X)$ coincide with equivalent norms.

The equivalence in Theorem 1.8 anwers the question proposed by Coifman and Weiss [12] mentioned in point (ii) above when $\mu(X)<\infty$. Furthermore if (A3) is satisfied with $\delta=1$, then one obtains the optimal range $\frac{n}{n+1}<p \leq 1$.

The final aim of our article is to apply Theorems 1.4 and 1.8 to certain differential operators on bounded/unbounded domains. We are able to prove the following new results:

(i) When $L$ is the second elliptic operator with Neumann boundary condition, we show that the Hardy spaces of extension or the Hard spaces of Coifman and Weiss $H_{C W}^{p}(\Omega)$ coincides with $H_{L, \text { max }}^{p}(\Omega)$ and $H_{L, \text { rad }}^{p}(\Omega)$. This not only extends the results in [3] to $p<1$, but also furnishes a new result even for $p=1$ with bounded $\Omega$. See Theorem 4.1.

(ii) When $L$ is a second elliptic operator with Dirichlet boundary condition, we show that the Hardy spaces of Miyachi $H_{M i}^{p}(\Omega)$ coincides with $H_{L, \max }^{p}(\Omega)$ and $H_{L, \text { rad }}^{p}(\Omega)$. Our paper is the first to give maximal function characterizations for the Hardy spaces of Miyachi $H_{M i}^{p}(\Omega)$. Furthermore, in the particular case when $\Omega$ is a strongly Lipschitz domain such that either $\Omega$ is bounded or $\Omega^{c}$ is unbounded, then we have $H_{r}^{p}(\Omega) \equiv H_{L, \text { max }}^{p}(\Omega) \equiv H_{L, \text { rad }}^{p}(\Omega)$, improving the results of [3] for $p<1$. Here $H_{r}^{p}(\Omega)$ is the Hardy spaces of restriction. See Theorem 4.4 .

(iii) When $L$ is a Schrödinger operators with Dirichlet boundary condition, we introduce a new version of Hardy space of Miyachi type $H_{\rho}^{p}(\Omega)$ and show that $H_{r}^{p}(\Omega) \equiv H_{L, \max }^{p}(\Omega) \equiv$ $H_{L, \text { rad }}^{p}(\Omega)$. See Theorem 4.11.

(iv) When $L$ is a Fourier-Bessel operator on $((0,1), d x)$ or $\left((0,1), x^{2 \nu+1} d x\right)$, we show that the maximal Hardy spaces $H_{L, \text { max }}^{p}(\Omega)$ and $H_{L \text {,rad }}^{p}(\Omega)$ enjoy certain atomic characterizations which extends the results in [19] to $p<1$ and a larger range of $\nu$. See Theorem 4.17 and Theorem 4.26.

We close this introduction with some remarks on the organization of the article. Section 2 collects some useful estimates for the operator $L$ arising from (A1) and (A2). Section 3 contain the proofs of Theorems 1.4 and 1.8, while the applications can be found in Section 4.

Notation. As usual we use $C$ and $c$ to denote positive constants that are independent of the main parameters involved but may differ from line to line. The notation $A \lesssim B$ denotes $A \leq C B$, and $A \sim B$ means that both $A \lesssim B$ and $B \lesssim A$ hold. We use $f_{E} f d \mu=\frac{1}{\mu(E)} \int_{E} f d \mu$ to denote the average of $f$ over $E$. We write $B(x, r)$ to denote the ball centred at $x$ with radius $r$. By a 'ball $B$ ' we mean the ball $B\left(x_{B}, r_{B}\right)$ with some fixed centre $x_{B}$ and radius $r_{B}$. The annuli around a given ball $B$ will be denoted by $S_{j}(B)=2^{j+1} B \backslash 2^{j} B$ for $j \geq 1$ and $S_{0}(B)=2 B$ for $j=0$. 


\section{Some KeRnel And maximal Function estimates}

Let $L$ satisfy (A1) and (A2). Denote by $E_{L}(\lambda)$ a spectral decomposition of $L$. Then by spectral theory, for any bounded Borel funtion $F:[0, \infty) \rightarrow \mathbb{C}$ we can define

$$
F(L)=\int_{0}^{\infty} F(\lambda) d E_{L}(\lambda)
$$

as a bounded operator on $L^{2}(X)$. It is well-known that the kernel $K_{\cos (t \sqrt{L})}$ of $\cos (t \sqrt{L})$ satisfies

$$
\operatorname{supp} K_{\cos (t \sqrt{L})} \subset\{(x, y) \in X \times X: d(x, y) \leq t\} .
$$

See for example [13]. We have the following useful lemmas.

Lemma 2.1 ([22]). Let $\varphi \in C_{0}^{\infty}(\mathbb{R})$ be an even function with $\operatorname{supp} \varphi \subset(-1,1)$ and $\int \varphi=2 \pi$. Denote by $\Phi$ the Fourier transform of $\varphi$. Then for every $k \in \mathbb{N}$, the kernel $K_{(t \sqrt{L})^{k} \Phi(t \sqrt{L})}(\cdot, \cdot)$ of $(t \sqrt{L})^{k} \Phi(t \sqrt{L})$ satisfies

$$
\operatorname{supp} K_{(t \sqrt{L})^{k} \Phi(t \sqrt{L})} \subset\{(x, y) \in X \times X: d(x, y) \leq t\},
$$

and

$$
\left|K_{(t \sqrt{L})^{k} \Phi(t \sqrt{L})}(x, y)\right| \leq \frac{C}{\mu(B(x, t))} .
$$

Lemma 2.2. (a) Let $\varphi \in \mathscr{S}(\mathbb{R})$ be an even function. Then for any $N>0$ there exists $C$ such that

$$
\left|K_{\varphi(t \sqrt{L})}(x, y)\right| \leq \frac{C}{\mu(B(x, t))+\mu(B(y, t))}\left(1+\frac{d(x, y)}{t}\right)^{-n-N},
$$

for all $t>0$ and $x, y \in X$.

(b) Let $\varphi_{1}, \varphi_{2} \in \mathscr{S}(\mathbb{R})$ be even functions. Then for any $N>0$ there exists $C$ such that

$$
\left|K_{\varphi_{1}(t \sqrt{L}) \varphi_{2}(s \sqrt{L})}(x, y)\right| \leq C \frac{1}{\mu(B(x, t))+\mu(B(y, t))}\left(1+\frac{d(x, y)}{t}\right)^{-n-N},
$$

for all $t \leq s<2 t$ and $x, y \in X$.

(c) Let $\varphi_{1}, \varphi_{2} \in \mathscr{S}(\mathbb{R})$ be even functions with $\varphi_{2}^{(\nu)}(0)=0$ for $\nu=0,1, \ldots, 2 \ell$ for some $\ell \in \mathbb{Z}^{+}$. Then for any $N>0$ there exists $C$ such that

$$
\left|K_{\varphi_{1}(t \sqrt{L}) \varphi_{2}(s \sqrt{L})}(x, y)\right| \leq C\left(\frac{s}{t}\right)^{2 \ell} \frac{1}{\mu(B(x, t))+\mu(B(y, t))}\left(1+\frac{d(x, y)}{t}\right)^{-n-N},
$$

for all $t \geq s>0$ and $x, y \in X$.

Proof. (a) The estimate (9) was proved in [6, Lemma 2.3] in the particular case $X=\mathbb{R}^{n}$ but the proof is still valid in the spaces of homogeneous type. For the items (b) and (c) we refer to $[4]$.

For any even function $\varphi \in \mathscr{S}(\mathbb{R}), \alpha>0$ and $f \in L^{2}(X)$ we define

$$
\varphi_{L, \alpha}^{*}(f)(x)=\sup _{t>0} \sup _{d(x, y)<\alpha t}|\varphi(t \sqrt{L}) f(y)|,
$$

and

$$
\varphi_{L, \alpha}^{+}(f)(x)=\sup _{t>0}|\varphi(t \sqrt{L}) f(x)| .
$$

As usual, we drop the index $\alpha$ when $\alpha=1$.

The following results are taken from Proposition 2.3 and Theorem 3.1 in [32], respectively. 
Proposition 2.3. Let $p \in(0,1]$. Let $\varphi_{1}, \varphi_{2} \in \mathbb{R}$ be even functions with $\varphi_{1}(0)=1$ and $\varphi_{2}(0)=0$ and $\alpha_{1}, \alpha_{2}>0$. Then for every $f \in L^{2}(X)$ we have

$$
\left\|\left(\varphi_{2}\right)_{L, \alpha_{2}}^{*} f\right\|_{L^{p}(X)} \lesssim\left\|\left(\varphi_{1}\right)_{L, \alpha_{1}}^{*} f\right\|_{L^{p}(X)} .
$$

As a consequence, for every even function $\varphi$ with $\varphi(0)=1$ and $\alpha>0$ we have

$$
\left\|\varphi_{L, \alpha}^{*} f\right\|_{L^{p}(X)} \sim\left\|f_{L}^{*}\right\|_{L^{p}(X)} .
$$

Proposition 2.4. Let $p \in(0,1]$. Let $\varphi \in \mathscr{S}(\mathbb{R})$ be an even function with $\varphi(0)=1$. Then we have

$$
\left\|\varphi_{L}^{*} f\right\|_{L^{p}(X)} \lesssim\left\|\varphi_{L}^{+} f\right\|_{L^{p}(X)},
$$

\section{Maximal function characterizations for Hardy spaces $H_{L}^{p}(X)$}

In this section we give the proofs of Theorems 1.4 and 1.8.

3.1. Proof of Theorem 1.4. By Proposition 2.4 we have $H_{L, \max }^{p}(X) \equiv H_{L, \text { rad }}^{p}(X)$ for $0<p \leq 1$ so it suffices to prove $H_{L, a t, M}^{p} \equiv H_{L, \max }^{p}$ for sufficiently large $M$. The direction $H_{L, a t, M}^{p}(X) \subset$ $H_{L, \text { max }}^{p}(X)$ follows by a similar argument to Step I in the proof of Theorem 3.5 of [15] provided $p \in(0,1]$ and $M>\frac{n}{2}\left(\frac{1}{p}-1\right)$. The rest of the proof is devoted to the remaining direction $H_{\text {max }, L}^{p} \subset H_{L, a t, M}^{p}(X)$.

Fix $f \in H_{L, \text { max }}^{p} \cap L^{2}(X)$. We shall show that $f$ has a $(p, M)_{L}$-representation $\sum_{j} \lambda_{j} a_{j}$ with $\left(\sum_{j}|\lambda|_{j}^{p}\right)^{1 / p} \lesssim\|f\|_{H_{L, \max }^{p}}$.

Let $\Phi$ be a function from Lemma 2.1. For $M \in \mathbb{N}, M>\frac{n}{2}\left(\frac{1}{p}-1\right)$ we have

$$
f=c_{\Phi, M} \int_{0}^{\infty}\left(t^{2} L\right)^{M} \Phi(t \sqrt{L}) \Phi(t \sqrt{L}) f \frac{d t}{t}
$$

in $L^{2}(X)$, where $c_{\Phi, M}=\left[\int_{0}^{\infty} x^{2 M} \Phi(x) \frac{d x}{x}\right]^{-1}$.

We now set

$$
\psi(x)=c_{\Phi, M} \int_{1}^{\infty}(t x)^{2 M} \Phi^{2}(t x) \frac{d t}{t}=c_{\Phi, M} \int_{x}^{\infty} t^{2 M} \Phi^{2}(t) \frac{d t}{t} .
$$

Then $\psi \in \mathscr{S}(\mathbb{R})$ and is an even function with $\psi(0)=1$. Moreover, for $s>0$,

$$
\psi(s x)=c_{\Phi, M} \int_{s}^{\infty}(t x)^{2 M} \Phi^{2}(t x) \frac{d t}{t} .
$$

This implies, for $s>0$,

$$
\psi(s \sqrt{L}) f=c_{\Phi, M} \int_{s}^{\infty}(t \sqrt{L})^{2 M} \Phi(t \sqrt{L}) \Phi(t \sqrt{L}) f \frac{d t}{t}
$$

in $L^{2}(X)$.

Setting $R_{0}=\operatorname{diam} X / 2$, we then decompose $f$ as follow

$$
\begin{aligned}
f & =c_{\Phi, M} \int_{0}^{R_{0}}\left(t^{2} L\right)^{M} \Phi(t \sqrt{L}) \Phi(t \sqrt{L}) f \frac{d t}{t}+c_{\Phi, M} \int_{R_{0}}^{\infty}\left(t^{2} L\right)^{M} \Phi(t \sqrt{L}) \Phi(t \sqrt{L}) f \frac{d t}{t} \\
& =c_{\Phi, M} \int_{0}^{R_{0}}\left(t^{2} L\right)^{M} \Phi(t \sqrt{L}) \Phi(t \sqrt{L}) f \frac{d t}{t}+\psi\left(R_{0} \sqrt{L}\right) f \\
& =: f_{1}+f_{2}
\end{aligned}
$$

in $L^{2}(X)$.

Define the maximal operator

$$
\mathbb{M}_{L} f(x)=\sup _{t>0} \sup _{d(x, y)<8 t}[|\psi(t \sqrt{L}) f(y)|+|\Phi(t \sqrt{L}) f(y)|] .
$$


Then Proposition 2.4 yields

$$
\left\|\mathbb{M}_{L} f\right\|_{L^{p}(X)} \lesssim\|f\|_{H_{\max , L}^{p}(X)} .
$$

Let us address the term $f_{2}$ first. Since $R_{0}=\operatorname{diam} X / 2$, we have, for all $x, y \in X$,

$$
\left|\psi\left(R_{0} \sqrt{L}\right) f(x)\right| \leq \sup _{d(z, y)<6 R_{0}}\left|\psi\left(R_{0} \sqrt{L}\right) f(z)\right| \leq \mathbb{M}_{L} f(y) .
$$

This implies

$$
\left\|f_{2}\right\|_{L^{\infty}(X)} \leq \inf _{y \in X} \mathbb{M}_{L} f(y) \leq \mu(X)^{-1 / p}\left\|\mathbb{M}_{L} f\right\|_{L^{p}(X)} \lesssim \mu(X)^{-1 / p}\|f\|_{H_{\max , L}^{p}(X)}
$$

where in the last inequality we used (17).

Therefore, we can write $f_{2}=\lambda a$ with $|\lambda| \lesssim\|f\|_{H_{\max , L}^{p}(X)}$ and $\|a\|_{L^{\infty}(X)} \leq \mu(X)^{-1 / p}$.

It remains to decompose the term $f_{1}$ in terms of $(p, M)_{L}$ atoms. To do this, for each $k \in \mathbb{Z}$ we set

$$
\Omega_{i}:=\left\{x \in X: \mathbb{M}_{L} f(x)>2^{i}\right\} .
$$

Since $\mathbb{M}_{L} f$ is lower-continuous and $X$ is bounded, there exists $i_{0}$ so that $\Omega_{i_{0}}=X$ and $\Omega_{i_{0}+1} \neq X$. Without loss of generality we may assume that $i_{0}=0$. Then for each $t>0$ we define

$$
\Omega_{i}^{t}= \begin{cases}\Omega_{0}, & i=0 \\ \left\{x: d\left(x, \Omega_{i}^{c}\right)>4 t\right\}, & i>0\end{cases}
$$

and $T_{i}^{t}=\Omega_{i}^{t} \backslash \Omega_{i+1}^{t}$

It is clear that $X=\bigcup_{i=0}^{\infty} T_{i}^{t}$ for each $t>0$. Hence,

$$
\begin{aligned}
f_{1} & =\sum_{i=0}^{\infty} c_{\Phi, M} \int_{0}^{R_{0}}\left(t^{2} L\right)^{M} \Phi(t \sqrt{L})\left[\Phi(t \sqrt{L}) f \cdot \chi_{T_{i}^{t}}\right] \frac{d t}{t} \\
& =: \sum_{i=0}^{\infty} f_{1}^{i} .
\end{aligned}
$$

We now consider $f_{1}^{0}$ first. For $x \in X$ we have

$$
f_{1}^{0}(x)=c_{\Phi, M} \int_{0}^{R_{0}} \int_{T_{0}^{t}} K_{\left(t^{2} L\right)^{M} \Phi(t \sqrt{L})}(x, y) \Phi(t \sqrt{L}) f(y) d \mu(y) \frac{d t}{t} .
$$

We now consider two cases: $x \in \Omega_{1}^{c}$ and $x \in \Omega_{1}$.

Case 1: $x \in \Omega_{1}^{c}$. In this situation, we can see that

$$
\operatorname{supp} K_{\left(t^{2} L\right)^{M} \Phi(t \sqrt{L})}(x, \cdot) \subset\{z: d(x, z)<t\} \subset T_{0}^{t}, \text { for all } t>0 .
$$

Therefore,

$$
\begin{aligned}
\left|f_{1}^{0}(x)\right| & =c_{\Phi, M} \int_{0}^{R_{0}} \int_{X} K_{\left(t^{2} L\right)^{M} \Phi(t \sqrt{L})}(x, y) \Phi(t \sqrt{L}) f(y) d \mu(y) \frac{d t}{t} \\
& =c_{\Phi, M} \int_{0}^{R_{0}}\left(t^{2} L\right)^{M} \Phi(t \sqrt{L}) \Phi(t \sqrt{L}) f(x) \frac{d t}{t} \\
& =\lim _{\epsilon \rightarrow 0} c_{\Phi, M} \int_{\epsilon}^{R_{0}}\left(t^{2} L\right)^{M} \Phi(t \sqrt{L}) \Phi(t \sqrt{L}) f(x) \frac{d t}{t} \\
& =\lim _{\epsilon \rightarrow 0} \psi(\epsilon \sqrt{L}) f(x)-\psi\left(R_{0} \sqrt{L}\right) f(x)
\end{aligned}
$$

where in the last inequality we used (15).

On the other hand, since $x \in \Omega_{1}^{c}$ we have $|\psi(s \sqrt{L}) f(x)| \leq 2$. This and (20) imply

$$
\left|f_{1}^{0}(x)\right| \leq 4, \quad \forall x \in \Omega_{1}^{c} .
$$


Case 2: $x \in \Omega_{1}$. To deal with this case, we write

$$
\begin{aligned}
f_{1}^{0}(x) & =c_{\Phi, M} \int_{0}^{R_{0}} \int_{T_{0}^{t}} K_{\left(t^{2} L\right)^{M} \Phi(t \sqrt{L})}(x, y) \Phi(t \sqrt{L}) f(y) d \mu(y) \frac{d t}{t} \\
& =\int_{0}^{d\left(x, \Omega_{1}^{c}\right) / 5} \ldots+\int_{d\left(x, \Omega_{1}^{c}\right) / 5}^{d\left(x, \Omega_{1}^{c}\right) / 3} \ldots+\int_{d\left(x, \Omega_{1}^{c}\right)}^{R_{0}} \ldots \\
& =: E_{1}(x)+E_{2}(x)+E_{3}(x) .
\end{aligned}
$$

For $t \in\left(0, d\left(x, \Omega_{1}^{c}\right) / 5\right)$ and $y \in T_{0}^{t}$ we have $d(x, y) \geq t$. This, along with Lemma 2.1, yields $K_{\left(t^{2} L\right)^{M} \Phi(t \sqrt{L})}(x, y)=0$, and hence $E_{1}(x)=0$.

For the second term, using Lemma 2.1 again we have

$$
\left|E_{2}(x)\right| \lesssim \int_{d\left(x, \Omega_{1}^{c}\right) / 3}^{d\left(x, \Omega_{1}^{c}\right) / 3} \sup _{y \in T_{0}^{t}}|\Phi(t \sqrt{L}) f(y)| \frac{d t}{t} .
$$

From the definition of the set $T_{0}^{t}$ it is easy to see that for each $y \in T_{0}^{t}$ we can find $z \in \Omega_{1}^{c}$ so that $d(y, z)<6 t$. For each such $z$ we have, since $z \in \Omega_{1}^{c}$,

$$
|\Phi(t \sqrt{L}) f(y)| \leq \mathbb{M}_{L} f(z) \leq 2 .
$$

Therefore we obtain $\left|E_{2}(x)\right| \lesssim 1$.

For the last term $E_{3}(x)$, we observe that for $t>d\left(x, \Omega_{1}^{c}\right) / 3$ and $x \in \Omega_{1}$ we have

$$
\operatorname{supp} K_{\left(t^{2} L\right)^{M} \Phi(t \sqrt{L})}(x, \cdot) \subset\{z: d(x, z)<t\} \subset T_{0}^{t} \text {, for all } t>0 .
$$

Hence, arguing similarly to (20) we come up with

$$
E_{3}(x)=\psi\left(s_{1} \sqrt{L}\right) f(x)-\psi\left(R_{0} \sqrt{L}\right) f(x)
$$

where $s_{1}=d\left(x, \Omega_{1}^{c}\right) / 3$.

Note that for $s>d\left(x, \Omega_{1}^{c}\right) / 3$ we can find $z \in \Omega_{1}^{c}$ so that $d(x, z)<3 s$. Hence,

$$
|\psi(t \sqrt{L}) f(x)| \leq \mathbb{M}_{L} f(z) \leq 2,
$$

since $z \in \Omega_{1}^{c}$.

As a consequence, we have $\left|E_{3}(x)\right| \lesssim 1$. We now take all estimates $E_{1}(x), E_{2}(x)$ and $E_{3}(x)$ into account to find that

$$
\left|f_{1}^{0}(x)\right| \lesssim 1, \quad \forall x \in \Omega_{1} .
$$

This, along with (21), implies that (22) in fact holds for every $x \in X$. Then we have

$$
\begin{aligned}
\left|f_{1}^{0}(x)\right| & \lesssim \mu(X)^{-1 / p} \mu\left(\Omega_{0}\right)^{1 / p} \\
& \lesssim \mu(X)^{-1 / p} \sum_{i=0}^{\infty} 2^{i} \mu\left(\Omega_{i}\right)^{1 / p} \sim \mu(X)^{-1 / p}\left\|\mathbb{M}_{L} f\right\|_{L^{p}(X)} \\
& \lesssim \mu(X)^{-1 / p}\|f\|_{H_{L, \max }^{p}(X)} .
\end{aligned}
$$

Hence, we can write $f_{1}^{0}=\lambda_{1}^{0} a_{1}^{0}$ so that $\left|\lambda_{1}^{0}\right| \lesssim\|f\|_{H_{\max , L}^{p}(X)}$ and $\left\|a_{1}^{0}\right\|_{L^{\infty}(X)} \leq \mu(X)^{-1 / p}$.

We now take care of the term $f_{1}^{i}$ with $i>0$. To do this, for each $i>0$ we apply a covering lemma in [12] (see also [14, Lemma 5.5]) to obtain a collection of balls $\left\{B_{i, k}:=B\left(x_{B_{i, k}}, r_{B_{i, k}}\right)\right.$ : $\left.x_{B_{i, k}} \in \Omega_{i}, r_{B_{i, k}}=d\left(x_{B_{i, k}}, \Omega_{i}^{c}\right) / 2, k=1, \ldots\right\}$ so that

(i) $\Omega_{i}=\cup_{k} B\left(x_{B_{i, k}}, r_{B_{i, k}}\right)$;

(ii) $\left\{B\left(x_{B_{i, k}}, r_{B_{i, k}} / 5\right)\right\}_{k=1}^{\infty}$ are disjoint. 
For each $i, k \in \mathbb{N}^{+}$and $t>0$ we set $B_{i, k}^{t}=B\left(x_{i, k}, r_{B_{i, k}}+2 t\right)$ which is a ball having the same center as $B_{i, k}$ with radius being $2 t$ greater than the radius of $B_{i, k}$. Then, for each $i, k \in \mathbb{N}^{+}$and $t>0$, we set

and

$$
R_{i, k}^{t}= \begin{cases}T_{i}^{t} \cap B_{i, k}^{t}, & \text { if } T_{i}^{t} \cap B_{i, k} \neq \emptyset \\ 0, & \text { if } T_{i}^{t} \cap B_{i, k}=\emptyset,\end{cases}
$$

$$
E_{i, k}^{t}=R_{i, k}^{t} \backslash \cup_{\ell>k} R_{i, k}^{t}
$$

It is easy to see that for each $i \in \mathbb{N}^{+}$and $t>0$ we have

$$
T_{i}^{t}=\bigcup_{k \in \mathbb{N}^{+}} E_{i, k}^{t}
$$

Hence, from (19) we have, for $i \in \mathbb{N}^{+}$,

$$
f_{1}^{i}=\sum_{k \in \mathbb{N}^{+}} c_{\Phi, M} \int_{0}^{R_{0}}\left(t^{2} L\right)^{M} \Phi(t \sqrt{L})\left[\Phi(t \sqrt{L}) f \cdot \chi_{E_{i, k}^{t}}\right] \frac{d t}{t}
$$

and set $a_{i, k}=0$ if $E_{i, k}^{t}=\emptyset$.

We now define $\lambda_{i, k}=2^{i} \mu\left(B_{i, k}\right)^{1 / p}$ and $a_{i, k}=L^{M} b_{i, k}$ where

$$
b_{i, k}=\frac{c_{\Phi, M}}{\lambda_{i, k}} \int_{0}^{R_{0}} t^{2 M} \Phi(t \sqrt{L})\left[\Phi(t \sqrt{L}) f \cdot \chi_{E_{i, k}^{t}}\right] \frac{d t}{t} .
$$

Then it can be seen that

$$
f_{1}=\sum_{i \in \mathbb{N}^{+}} f_{1}^{i}=\sum_{i, k \in \mathbb{N}^{+}} \lambda_{i, k} a_{i, k}
$$

in $L^{2}(X)$; moreover,

$$
\sum_{i, k \in \mathbb{N}^{+}}\left|\lambda_{i, k}\right|^{p}=\sum_{i, k \in \mathbb{N}^{+}} 2^{i p} \mu\left(B_{i, k}\right) \lesssim \sum_{i \in \mathbb{N}^{+}} 2^{i p} \mu\left(\Omega_{i}\right) \lesssim\left\|\mathbb{M}_{L} f\right\|_{L^{p}(X)}^{p} \lesssim\|f\|_{H_{L, \max }^{p}(X)}^{p} .
$$

Therefore, it suffices to prove that each $a_{i, k} \neq 0$ is a $(p, M)_{L}$ atom associated to the ball $B_{i, k}^{*}:=8 B_{i, k}$. Indeed, if $r_{B_{i, k}}<t / 2$, then we have $d\left(x_{B_{i, k}}, \Omega_{i}^{c}\right)=2 r_{B_{i, k}}<t$. Therefore,

$$
B_{i, k}^{t}=B\left(x_{B_{i, k}}, r_{B_{i, k}}+2 t\right) \subset\left\{x: d\left(x, \Omega_{i}^{c}\right)<4 t\right\} .
$$

This implies that $R_{i, k}^{t}:=T_{i}^{t} \cap B_{i, k}^{t}=\emptyset$. Hence, if $a_{i, k} \neq 0$, then $r_{B_{i, k}} \geq t / 2$. This, along with (24) and Lemma 2.1, implies that

$$
\operatorname{supp} L^{m} b_{i, k} \subset B_{i, k}^{*}, \quad \forall m=0,1, \ldots, M .
$$

It remains to show that

$$
\left|L^{m} b_{i, k}\right|_{L^{\infty}(X)} \lesssim r_{B_{i, k}}^{2(M-m)} \mu\left(B_{i, k}\right)^{-1 / p}, \quad \forall m=0,1, \ldots, M .
$$

For $m=0,1, \ldots, M-1$, since $r_{B_{i, k}} \geq t / 2$ as $a_{i, k} \neq 0$, we have

$$
\left|L^{m} b_{i, k}(x)\right| \leq \frac{c_{\Phi, M}}{\lambda_{i, k}} \int_{0}^{2 r_{B_{i, k}}} t^{2(M-m)}\left|\left(t^{2} L\right)^{m} \Phi(t \sqrt{L})\left[\Phi(t \sqrt{L}) f \cdot \chi_{E_{i, k}^{t}}\right](x)\right| \frac{d t}{t} .
$$

This along with Lemma 2.1 implies that

$$
\begin{aligned}
\left|L^{m} b_{i, k}(x)\right| & \lesssim \frac{1}{\lambda_{i, k}} \int_{0}^{2 r_{B_{i, k}}} t^{2(M-m)} \int_{E_{i, k}^{t}}|\Phi(t \sqrt{L}) f(y)| d \mu(y) \frac{d t}{t} \\
& \lesssim \frac{1}{\lambda_{i, k}} \int_{0}^{2 r_{B_{i, k}}} t^{2(M-m)} \int_{T_{i}^{t}}|\Phi(t \sqrt{L}) f(y)| d \mu(y) \frac{d t}{t} .
\end{aligned}
$$

Note that for each $y \in T_{i}^{t}$ there exists $z \in \Omega_{i+1}^{c}$ so that $d(y, z)<4 t$. Hence,

$$
|\Phi(t \sqrt{L}) f(y)| \leq \mathbb{M}_{L} f(z) \leq 2^{i+1}, \quad \forall y \in T_{i}^{t} .
$$


Therefore, for all $m=0,1, \ldots, M-1$,

$$
\left|L^{m} b_{i, k}(x)\right| \lesssim \frac{2^{i}}{\lambda_{i, k}} \int_{0}^{2 r_{B_{i, k}}} t^{2(M-m)} \frac{d t}{t} \lesssim \mu\left(B_{i, k}\right)^{-1 / p} .
$$

For $m=M$, we have

$$
L^{M} b_{i, k}(x)=\frac{c_{\Phi, M}}{\lambda_{i, k}} \int_{0}^{2 r_{B_{i, k}}}\left(t^{2} L\right)^{M} \Phi(t \sqrt{L})\left[\Phi(t \sqrt{L}) f \cdot \chi_{E_{i, k}^{t}}\right](x) \frac{d t}{t} .
$$

From (23) we have

$$
E_{i, k}^{t}=\left(T_{i}^{t} \cap B_{i, k}^{t}\right) \backslash\left(T_{i}^{t} \cap F_{i, k}^{t}\right)
$$

where $F_{i, k}^{t}:=\cup_{\ell>k} B_{i, \ell}^{t}=\left\{x: d\left(x, \cup_{\ell>k} B_{i, \ell}\right)<2 t\right\}$.

Hence, we can rewrite

$$
\begin{aligned}
L^{M} b_{i, k}(x)= & \frac{c_{\Phi, M}}{\lambda_{i, k}} \int_{0}^{2 r_{B_{i, k}}} \int_{T_{i}^{t} \cap B_{i, k}^{t}} K_{\left(t^{2} L\right)^{M} \Phi(t \sqrt{L})}(x, y) \Phi(t \sqrt{L}) f(y) d \mu(y) \frac{d t}{t} \\
& -\frac{c_{\Phi, M}}{\lambda_{i, k}} \int_{0}^{2 r_{B_{i, k}}} \int_{T_{i}^{t} \cap F_{i, k}^{t} \cap B_{i, k}^{t}} K_{\left(t^{2} L\right)^{M} \Phi(t \sqrt{L})}(x, y) \Phi(t \sqrt{L}) f(y) d \mu(y) \frac{d t}{t} \\
= & \frac{c_{\Phi, M}}{\lambda_{i, k}} I_{i, k}(x)+\frac{c_{\Phi, M}}{\lambda_{i, k}} J_{i, k}(x) .
\end{aligned}
$$

We have the following result whose proof will be given after the proof of the theorem.

Lemma 3.1. There exists a constant $C>0$ so that for all $i, k \in \mathbb{N}^{+}$and $x \in X$ we have

$$
\left|I_{i, k}(x)\right|+\left|J_{i, k}(x)\right| \lesssim 2^{i},
$$

where $I_{i, k}$ and $J_{i, k}$ have been defined in (25).

We now just substitute (26) into (25) to conclude that

$$
\left|L^{M} b_{i, k}(x)\right| \lesssim \mu\left(B_{i, k}\right)^{-1 / p} .
$$

which concludes the proof of Theorem 1.4.

We now give the proof for Lemma 3.1.

Proof of Lemma 3.1: For any subset $U$ of $X$ and for each $t>0$ we define $U^{t}:=\{x: d(x, U)<$ $2 t\}$. Now let $U$ and $V$ be any two subsets of $X$. For each $s \in\left(0, R_{0}\right]$ and $i \in \mathbb{N}^{+}$we define

$$
\begin{gathered}
g_{s}(x)=c_{\Phi, M} \int_{0}^{s}\left(t^{2} L\right)^{M} \Phi(t \sqrt{L})\left[\Phi(t \sqrt{L}) f \cdot \chi_{T_{i}^{t}}\right] \frac{d t}{t}, \quad x \in X . \\
g_{U, s}(x)=c_{\Phi, M} \int_{0}^{s}\left(t^{2} L\right)^{M} \Phi(t \sqrt{L})\left[\Phi(t \sqrt{L}) f \cdot \chi_{T_{i}^{t} \cap U^{t}}\right] \frac{d t}{t}, \quad x \in X .
\end{gathered}
$$

and

$$
g_{U, V, s}(x)=c_{\Phi, M} \int_{0}^{s}\left(t^{2} L\right)^{M} \Phi(t \sqrt{L})\left[\Phi(t \sqrt{L}) f \cdot \chi_{T_{i}^{t} \cap U^{t} \cap V^{t}}\right] \frac{d t}{t}, \quad x \in X .
$$

We claim that (26) is a consequence of the following three estimates.

$$
\begin{gathered}
\left|g_{s}(x)\right| \lesssim 2^{i}, \\
\left|g_{U, s}(x)\right| \lesssim 2^{i}, \\
\left|g_{U, V, s}(x)\right| \lesssim 2^{i},
\end{gathered}
$$

for any $U, V \subset X$ and $s \in\left(0, R_{0}\right], i \in \mathbb{N}^{+}$and $x \in X$

Indeed by firstly applying (28) for $U=B_{i, k}$ we obtain $\left|I_{i, k}(x)\right| \lesssim 2^{i}$ for all $x \in X$. Secondly by applying (29) for $U=B_{i, k}$ and $V=F_{i, k}$ we get $\left|J_{i, k}(x)\right| \lesssim 2^{i}$. Thus (26) holds.

It remains to show (27)-(28).

We begin with (27). Indeed, we now consider two cases: $x \in \Omega_{i+1}^{c}$ and $x \in \Omega_{i+1}$. 
Case 1: $x \in \Omega_{i+1}^{c}$. There are two subcases $s>d\left(x, \Omega_{i}^{c}\right) / 3$ and $s \leq d\left(x, \Omega_{i}^{c}\right) / 3$. We just consider the first case, since the latter is similar and even easier.

We write

$$
\begin{aligned}
g_{s}(x)= & c_{\Phi, M} \int_{0}^{d\left(x, \Omega_{i}^{c}\right) / 5} \int_{T_{i}^{t}} K_{\left(t^{2} L\right)^{M} \Phi(t \sqrt{L})}(x, y) \Phi(t \sqrt{L}) f(y) d \mu(y) \frac{d t}{t} \\
& +c_{\Phi, M} \int_{d\left(x, \Omega_{i}^{c}\right) / 5}^{d\left(x, \Omega_{i}^{c}\right) / 3} \int_{T_{i}^{t}} K_{\left(t^{2} L\right)^{M} \Phi(t \sqrt{L})}(x, y) \Phi(t \sqrt{L}) f(y) d \mu(y) \frac{d t}{t} \\
& +c_{\Phi, M} \int_{d\left(x, \Omega_{i}^{c}\right) / 3}^{s} \int_{T_{i}^{t}} K_{\left(t^{2} L\right)^{M} \Phi(t \sqrt{L})}(x, y) \Phi(t \sqrt{L}) f(y) d \mu(y) \frac{d t}{t} \\
= & : A_{1}(x)+A_{2}(x)+A_{3}(x) .
\end{aligned}
$$

For the first term, we can see that $B(x, t) \subset T_{i}^{t}$ as $t \in\left(0, d\left(x, \Omega_{i}^{c}\right) / 5\right)$. Hence, by Lemma 2.1 we find that

$$
\begin{aligned}
A_{1}(x) & =c_{\Phi, M} \int_{0}^{d\left(x, \Omega_{i}^{c}\right) / 5} \int_{X} K_{\left(t^{2} L\right)^{M} \Phi(t \sqrt{L})}(x, y) \Phi(t \sqrt{L}) f(y) d \mu(y) \frac{d t}{t} \\
& =c_{\Phi, M} \int_{0}^{d\left(x, \Omega_{i}^{c}\right) / 5}\left(t^{2} L\right)^{M} \Phi(t \sqrt{L}) \Phi(t \sqrt{L}) f(x) \frac{d t}{t} \\
& =\lim _{\epsilon \rightarrow 0} \psi(\epsilon \sqrt{L}) f(x)-\psi\left(s_{2} \sqrt{L}\right) f(x)
\end{aligned}
$$

where $s_{2}=d\left(x, \Omega_{i}^{c}\right) / 5$.

Note that $x \in \Omega_{i+1}^{c}$. Hence, $|\psi(t \sqrt{L}) f(x)| \leq 2^{i+1}$ for all $t>0$. As a consequence, we have $A_{1}(x) \leq 2^{i+1}$.

For the second term $A_{2}(x)$, using Lemma 2.1 we obtain

$$
\left|A_{2}(x)\right| \lesssim \int_{d\left(x, \Omega_{i}^{c}\right) / 5}^{d\left(x, \Omega_{i}^{c}\right) / 3} \sup _{y \in T_{i}^{t}}|\Phi(t \sqrt{L}) f(y)| \frac{d t}{t} .
$$

Moreover, since $B(y, 8 t) \cap \Omega_{i+1}^{c} \neq \emptyset$ as $y \in T_{i}^{t}$ and $d\left(x, \Omega_{i}^{c}\right) / 5 \leq t \leq d\left(x, \Omega_{i}^{c}\right) / 3$, we have

$$
\sup _{y \in T_{i}^{t}}|\Phi(t \sqrt{L}) f(y)| \leq 2^{i+1}, \quad \forall d\left(x, \Omega_{i}^{c}\right) / 5 \leq t \leq d\left(x, \Omega_{i}^{c}\right) / 3 .
$$

Hence, $A_{2}(x) \lesssim 2^{i}$.

The last term $A_{3}(x)$ is zero, since in this situation we have $B(x, t) \cap T_{i}^{t}=\emptyset$. Gathering all estimates of $A_{1}(x), A_{2}(x)$ and $A_{3}(x)$ we arrive at $\left|g_{s}(x)\right| \lesssim 2^{i}$.

Case 2: $x \in \Omega_{i+1}$. We only consider the case $s>d\left(x, \Omega_{i+1}^{c}\right)$. The case $s \leq d\left(x, \Omega_{i}^{c}\right)$ can be done similarly.

In this case, firstly we consider situation $d\left(x, \Omega_{i+1}^{c}\right) / 3<d\left(x, \Omega_{i}^{c}\right) / 4$. Then we split the integral in the expression of $g_{s}$ as follows

$$
\begin{aligned}
g_{s}(x)= & \int_{0}^{d\left(x, \Omega_{i+1}^{c}\right) / 5} \ldots+\int_{d\left(x, \Omega_{i+1}^{c}\right) / 5}^{d\left(x, \Omega_{i+1}^{c}\right) / 3} \cdots+\int_{d\left(x, \Omega_{i+1}^{c}\right) / 3}^{d\left(x, \Omega_{i}^{c}\right) / 4} \cdots \\
& +\int_{d\left(x, \Omega_{i}^{c}\right) / 4}^{d\left(x, \Omega_{i}^{c}\right) / 3} \cdots+\int_{d\left(x, \Omega_{i}^{c}\right) / 3}^{s} \cdots=: \sum_{\ell=1}^{5} B_{\ell}(x) .
\end{aligned}
$$

Arguing as in the first case, we have $B_{1}(x)=B_{5}(x)=0$. The terms $B_{2}(x)$ and $B_{4}(x)$ can be dealt with in a similar way to $A_{2}(x)$ so that

$$
\left|B_{2}(x)\right|+\left|B_{4}(x)\right| \lesssim 2^{i} .
$$

For the term $B_{3}(x)$, we note that by Lemma 2.1 we have,

$$
\operatorname{supp} K_{\left(t^{2} L\right)^{M} \Phi(t \sqrt{L})}(x, y)(x, \cdot) \subset B(x, t) \subset T_{i}^{t}, \quad d\left(x, \Omega_{i+1}^{c}\right) / 3<t<d\left(x, \Omega_{i}^{c}\right) / 4 .
$$


At this stage, arguing similarly to the estimate of $A_{1}(x)$ in Case 1 , we find that

$$
\left|B_{3}(x)\right| \lesssim 2^{i}
$$

If $d\left(x, \Omega_{i+1}^{c}\right) / 3 \geq d\left(x, \Omega_{i}^{c}\right) / 4$, we split the integral in the expression of $g_{s}$ as follows:

$$
g_{s}(x)=\int_{0}^{d\left(x, \Omega_{i+1}^{c}\right) / 5} \ldots+\int_{d\left(x, \Omega_{i+1}^{c}\right) / 5}^{d\left(x, \Omega_{i}^{c}\right) / 4} \ldots+\int_{d\left(x, \Omega_{i}^{c}\right) / 4}^{d\left(x, \Omega_{i}^{c}\right) / 3} \ldots+\int_{d\left(x, \Omega_{i}^{c}\right) / 3}^{s} \ldots
$$

Then we use the argument as above to dominate $\left|g_{s}(x)\right|$ by a multiple of $2^{i}$. Hence, this completes the proof of $(27)$.

We turn to the proof of (28).

Now if $x \in U$, then

$$
\operatorname{supp} K_{\left(t^{2} L\right)^{M} \Phi(t \sqrt{L})}(x, y)(x, \cdot) \subset B(x, t) \subset U^{t}, \quad \forall t>0 .
$$

Hence, $g_{U, s}(x)=g_{s}(x)$, and by $(27)$ we have $\left|g_{U, s}(x)\right| \lesssim 2^{i}$.

Otherwise, if $x \notin U$, there two cases $s>d\left(x, U^{c}\right)$ and $s \leq d\left(x, U^{c}\right)$. We will only consider the first case $s>d\left(x, U^{c}\right)$, since the second case can be done similarly. Assuming $s>d\left(x, U^{c}\right)$, we now break $g_{U, s}$ into 3 terms

$$
\begin{aligned}
g_{U, s}(x)= & c_{\Phi, M} \int_{0}^{d\left(x, U^{c}\right) / 3} \int_{T_{i}^{t} \cap U^{t}} K_{\left(t^{2} L\right)^{M} \Phi(t \sqrt{L})}(x, y) \Phi(t \sqrt{L}) f(y) d \mu(y) \frac{d t}{t} \\
& +c_{\Phi, M} \int_{d\left(x, U^{c}\right) / 3}^{d\left(x, U^{c}\right)} \int_{T_{i}^{t} \cap U^{t}} K_{\left(t^{2} L\right)^{M} \Phi(t \sqrt{L})}(x, y) \Phi(t \sqrt{L}) f(y) d \mu(y) \frac{d t}{t} \\
& +c_{\Phi, M} \int_{d\left(x, U^{c}\right)}^{s} \int_{T_{i}^{t} \cap U^{t}} K_{\left(t^{2} L\right)^{M} \Phi(t \sqrt{L})}(x, y) \Phi(t \sqrt{L}) f(y) d \mu(y) \frac{d t}{t} \\
= & D_{1}(x)+D_{2}(x)+D_{3}(x) .
\end{aligned}
$$

The first term $D_{1}(x)=0$, since

$$
\operatorname{supp} K_{\left(t^{2} L\right)^{M} \Phi(t \sqrt{L})}(x, y)(x, \cdot) \subset B(x, t) \subset\left(U^{t}\right)^{c} .
$$

The second term can be estimated similarly to the term $A_{2}(x)$ so that $\left|D_{2}(x)\right| \lesssim 2^{i}$.

For the last term, we note that as $t>d\left(x, U^{c}\right)$ we have

$$
\operatorname{supp} K_{\left(t^{2} L\right)^{M} \Phi(t \sqrt{L})}(x, y)(x, \cdot) \subset B(x, t) \subset U^{t} .
$$

Hence,

$$
D_{3}(x)=c_{\Phi, M} \int_{d\left(x, U^{c}\right)}^{s} \int_{T_{i}^{t}} K_{\left(t^{2} L\right)^{M} \Phi(t \sqrt{L})}(x, y) \Phi(t \sqrt{L}) f(y) d \mu(y) \frac{d t}{t}=g_{s}(x)-g\left(s_{3}\right)(x)
$$

where $s_{3}=d\left(x, U^{c}\right) \leq R_{0}$.

Using (27) we obtain $\left|D_{3}(x)\right| \lesssim 2^{i}$ and this proves (28).

The estimate (29) can be done by repeating the argument for (28). This completes the proof of Lemma 3.1.

Remark 3.2. The argument in the proof of Theorem 1.4 also shows that the result in Theorem 1.4 is still true if we define the non-tangential maximal function and the radial maximal function by

$$
f_{L}^{*}(x)=\sup _{0<t<\operatorname{diam}^{2} X} \sup _{d(x, y)<t}\left|e^{-t L} f(y)\right|
$$

and

$$
f_{L}^{+}(x)=\sup _{0<t<\operatorname{diam}^{2} X}\left|e^{-t L} f(x)\right|,
$$

respectively. 
3.2. Proof of Theorem 1.8. Due to the validity of Theorem 1.4, we need only to show that $H_{C W}^{p}(X) \equiv H_{L, \text { rad }}^{p}(X)$ for $\frac{n}{n+\delta}<p \leq 1$. Now the inclusion $H_{C W}^{p}(X) \hookrightarrow H_{L, \text { rad }}^{p}(X)$ is standard and will be omitted. For the reverse inclusion, since $H_{L, \text { rad }}^{p}(X) \equiv H_{L, a t, M}^{p}(X)$, again from Theorem 1.4, it suffices to prove $H_{L, a t, M}^{p}(X) \hookrightarrow H_{C W}^{p}(X)$.

Now if $a$ is a $(p, M)_{L}$-atom associated to a ball $B$, then there are two cases. If $a=\mu(X)^{-1 / p}$ then $a$ is also a $p$-atom and hence $a \in H_{C W}^{p}(X)$. Otherwise, we have $a=L^{M} b$ and by using (A4) and arguing similarly to the proof of Lemma 9.1 in [22] we have

$$
\int a(x) d \mu(x)=0 \text {. }
$$

Moreover parts (ii) and (iii) of Definition 1.1 shows that supp $a \subset B$ and $\|a\|_{L^{\infty}} \leq \mu(B)^{-1 / p}$. It follows that $a$ is also a $p$-atom and hence $a \in H_{C W}^{p}(X)$. As a consequence, $H_{L, a t, M}^{p}(X) \hookrightarrow$ $H_{C W}^{p}(X)$ and this completes our proof of Theorem 1.8.

\section{Applications}

In this section we apply Theorems 1.4 and 1.8 to give maximal characterizations of atomic Hardy spaces for various differential operators on domains.

4.1. Second-order elliptic operators with Neumann boundary conditions. Let $A$ : $\mathbb{R}^{n} \rightarrow M_{n}(\mathbb{R})$ be a real matrix-valued function and define

$$
\|A\|_{\infty}=\sup _{x \in \mathbb{R}^{n},|\xi|=|\eta|=1}|A(x) \xi \cdot \eta| .
$$

We assume that $A$ is symmetric and satisfies the following conditions for all $x \in \mathbb{R}^{n}, \xi \in \mathbb{R}^{n}$ :

$$
\|A\|_{\infty} \leq \Lambda^{-1} \text { and } A(x) \xi \cdot \xi \geq \Lambda|\xi|^{2}
$$

for some $\Lambda>0$.

Let $\Omega$ be an connected open bounded/unbounded domain in $\mathbb{R}^{n}$ satisfying a doubling property. We do not assume any smoothness assumption on the boundary of $\Omega$ unless it is implied by other assumptions. Denote by $L_{N}$ the maximal-accretive operator on $L^{2}(\Omega)$ with largest domain $D\left(L_{N}\right) \subset W^{1,2}(\Omega)$ such that

$$
\left\langle L_{N} f, g\right\rangle=\int_{\Omega} A \nabla f \cdot \nabla g, \quad \forall f \in D\left(L_{N}\right), \quad g \in W^{1,2}(\Omega) .
$$

We then have the following.

Theorem 4.1. Assume that the kernel $p_{t}(x, y)$ of $e^{-t L_{N}}$ satisfies the following conditions:

(N1) There exists $C, C>0$ so that

$$
\left|p_{t}(x, y)\right| \leq \frac{C}{\mu\left(B_{\Omega}(x, \sqrt{t})\right)} \exp \left(-\frac{|x-y|^{2}}{c t}\right)
$$

for all $0<t<\operatorname{diam} X$ and $x, y \in \Omega$, where $B_{\Omega}(x, r)=B(x, r) \cap \Omega$.

(N2) There exist $\gamma \in(0,1]$ and $C, c>0$ so that

$$
\left|p_{t}(x, y)-p_{t}\left(x, y^{\prime}\right)\right| \leq\left(\frac{\left|y-y^{\prime}\right|}{\sqrt{t}}\right)^{\gamma} \frac{C}{\mu\left(B_{\Omega}(x, \sqrt{t})\right)} \exp \left(-\frac{|x-y|^{2}}{c t}\right)
$$

for all $0<t<\operatorname{diam} \Omega$ and $x, y, y^{\prime} \in \Omega$ so that $\left|y-y^{\prime}\right|<\sqrt{t} / 2$.

Then we have

$$
H_{L_{N}, \max }^{p}(\Omega) \equiv H_{L_{N}, \mathrm{rad}}^{p}(\Omega) \equiv H_{C W}^{p}(\Omega), \quad \frac{n}{n+\gamma}<p \leq 1 .
$$

Remark 4.2. We note that when $\Omega$ is a strongly Lipschitz domain, the equivalence (32) was obtained in [3] for $p=1$ and $|\Omega|=\infty$. Hence, in the case $|\Omega|<\infty$ our result is new even for $p=1$. 
Proof of Theorem 4.1. We need only to prove the case $|\Omega|<\infty$, since the case $|\Omega|=\infty$ is similar and easier. Now it is well-known that for every $x \in \Omega$ and $t>0$, we have

$$
\int_{\Omega} p_{t}(x, y) d \mu(y)=1 .
$$

Therefore, $L_{N}$ satisfies (A1)-(A4) and we may invoke Theorem 1.8 to conclude our proof.

4.2. Second-order elliptic operators with Dirichlet boundary conditions. Let $A$ and $\Omega$ be as in Subsection 4.1. Denote by $L_{D}$ the maximal-accretive operator on $L^{2}(\Omega)$ with largest domain $D\left(L_{D}\right) \subset W_{0}^{1,2}(\Omega)$ such that

$$
\left\langle L_{D} f, g\right\rangle=\int_{\Omega} A \nabla f \cdot \nabla g, \quad \forall f \in D\left(L_{D}\right), \quad g \in W_{0}^{1,2}(\Omega) .
$$

We shall consider the atomic spaces defined by Miyachi [29].

Definition 4.3 (Hardy spaces of Miyachi). Let $p \in(0,1]$. A bounded, measurable function $a: \Omega \rightarrow \mathbb{R}$ is called an $H_{M i}^{p}(\Omega)$-atom if

(i) $a$ is supported in a ball $B \subset \Omega$;

(ii) $\|a\|_{L^{\infty}(\Omega)} \leq|B|^{-1 / p}$;

(iii) either $2 B \subset \Omega$ and $4 B \cap \partial \Omega \neq \emptyset$, or $4 B \subset \Omega$ and

$$
\int x^{\alpha} a(x) d x=0
$$

for all multi-indices $\alpha$ with $|\alpha| \leq[n(1 / p-1)]$.

The Hardy space $H_{M i}^{p}(\Omega)$ is defined as the set of all $f \in \mathscr{S}^{\prime}$ such that

$$
f=\sum_{j} \lambda_{j} a_{j}
$$

where $a_{j}$ are $H_{M i}^{p}(\Omega)$-atoms and $\lambda_{j}$ are scalars with $\sum_{j}\left|\lambda_{j}\right|^{p}<\infty$. We also set

$$
\|f\|_{H_{M i}^{p}(\Omega)}^{p}=\inf \left\{\sum_{j}\left|\lambda_{j}\right|^{p}: f=\sum_{j} \lambda_{j} a_{j}\right\}
$$

where the infimum is taken over all such decompositions.

Our main result in this section is the following:

Theorem 4.4. Assume that the kernel $p_{t}(x, y)$ of $e^{-t L_{D}}$ satisfies the following conditions:

(D1) There exists $C, C>0$ so that

$$
\left|p_{t}(x, y)\right| \leq \frac{C}{\mu\left(B_{\Omega}(x, \sqrt{t})\right)} \exp \left(-\frac{|x-y|^{2}}{c t}\right)
$$

for all $t>0$ and $x, y \in \Omega$, where $B_{\Omega}(x, r)=B(x, r) \cap \Omega$.

(D2) There exist $\gamma \in(0,1]$ and $C, c>0$ so that

$$
\left|p_{t}(x, y)-p_{t}\left(x, y^{\prime}\right)\right| \leq\left(\frac{\left|y-y^{\prime}\right|}{\sqrt{t}}\right)^{\gamma} \frac{C}{\mu\left(B_{\Omega}(x, \sqrt{t})\right)} \exp \left(-\frac{|x-y|^{2}}{c t}\right)
$$

for all $0<t<\operatorname{diam} \Omega, x, y, y^{\prime} \in \Omega$ so that $\left|y-y^{\prime}\right|<\sqrt{t} / 2$.

Then we have

$$
H_{L_{D}, \max }^{p}(\Omega) \equiv H_{L_{D}, \mathrm{rad}}^{p}(\Omega) \equiv H_{M i}^{p}(\Omega), \quad \frac{n}{n+\gamma}<p \leq 1 .
$$

Remark 4.5. Some comments are in order.

(a) In the particular case when $\Omega$ is $\mathbb{R}^{n}$ or Lipschitz domains, the conditions (D1) and (D2) are always satisfied. See [3]. 
(b) Let $\phi \in C_{c}^{\infty}(B(0,1))$ be a non-negative radial function such that $\int \phi(x) d x=1$. It was proved in [29] that the Hardy spaces $H_{M i}^{p}(\Omega)$ can be characterized in terms of maximal functions of the form

$$
f^{+}(x)=\max _{0<t<\delta(x) / 2}\left|\phi_{t} * f(x)\right|
$$

where $\delta(x)=d\left(x, \Omega^{c}\right)$ and $\phi_{t}(x)=t^{-n} \phi(x / t)$. More precisely, we have

$$
\|f\|_{H_{M i}^{p}(\Omega)} \sim\left\|f^{+}\right\|_{L^{p}(\Omega)}, \quad 0<p \leq 1 .
$$

In this sense, our results give new maximal characterizations for the Hardy spaces $H_{M i}^{p}(\Omega)$.

(c) The Hardy space $H_{M i}^{p}(\Omega)$ is closely related to the Hardy space $H_{r}^{p}(\Omega)$ defined by

$$
H_{r}^{p}(\Omega)=\left\{f \in \mathscr{S}^{\prime}: \text { there exists } F \in H^{p}\left(\mathbb{R}^{n}\right) \text { so that }\left.F\right|_{\Omega}=f\right\}
$$

with the norm

$$
\|f\|_{H_{r}^{p}(\Omega)}=\inf \left\{\|F\|_{H_{r}^{p}\left(\mathbb{R}^{n}\right)}: F \in H^{p}\left(\mathbb{R}^{n}\right),\left.F\right|_{\Omega}=f\right\} .
$$

It is well-known that if $\Omega$ is a strongly Lipschitz domain such that either $\Omega$ is bounded or $\Omega^{c}$ is unbounded (see Subsection 4.3 for the precise definition), then $H_{M i}^{p}(\Omega) \equiv H_{r}^{p}(\Omega)$ for $0<p \leq 1$. See for instance [9]. For such domains, Theorem 4.4 implies that $H_{L, \mathrm{rad}}^{p}(\Omega) \equiv$ $H_{L, \max }^{p}(\Omega) \equiv H_{r}^{p}(\Omega)$. This gives a positive answer to the open question in [15] (mentioned in Section 5.1 of that article).

Before coming to the proof of Theorem 4.4, we need the following two technical results.

Lemma 4.6. Let $x_{0} \in \Omega$ and $r>0$ so that $B\left(x_{0}, 2 r\right) \subset \Omega$. Then we have

$$
\left|\int_{B\left(x_{0}, 2 r\right)} q_{t}(x, y) d x\right| \lesssim \frac{\sqrt{t}}{r} e^{-\frac{r^{2}}{c t}}
$$

for all $t>0$ and all $y \in B\left(x_{0}, r\right)$ where $q_{t}(x, y)$ is the kernel of $t L_{D} e^{-t L_{D}}$.

Proof. Let $\psi \in C_{c}^{\infty}\left(x_{0}, 2 r\right)$ so that $\psi=1$ on $B\left(x_{0}, 3 r / 2\right)$ and $|\nabla \psi| \lesssim 1 / r$. Then we have

$$
\int_{B\left(x_{0}, 2 r\right)} q_{t}(x, y) d x=\int_{B\left(x_{0}, 2 r\right)} q_{t}(x, y) \psi(x) d x+\int_{B\left(x_{0}, 2 r\right)} q_{t}(x, y)[1-\psi(x)] d x=: I_{1}+I_{2} .
$$

The Gaussian upper bound of $q_{t}(x, y)$ and the support condition of $(1-\psi)$ gives

$$
\left|I_{2}\right| \lesssim \exp \left(-\frac{r^{2}}{c^{\prime} t}\right) \int_{B\left(x_{0}, 2 r\right) \backslash B\left(x_{0}, 3 r / 2\right)} \frac{1}{t^{n / 2}} \exp \left(-\frac{|x-y|^{2}}{2 c t}\right) d x \lesssim \frac{\sqrt{t}}{r} e^{-\frac{r^{2}}{c^{\prime} t}} .
$$

For the term $I_{1}$ we first note that $q_{t}(x, y)=t L_{D}\left[p_{t}(\cdot, y)\right](x)$ and $p_{t}(\cdot, y) \in D\left(L_{D}\right)$. See for example [30]. Hence, from (33) and (30), coupled with the support property of $\nabla \psi$, we have

$$
\begin{aligned}
\left|I_{1}\right| & =t\left|\int_{\Omega} A \nabla_{x} p_{t}(x, y) \cdot \nabla \psi(x) d x\right| \\
& \lesssim \frac{t}{r} \int_{B\left(x_{0}, 2 r\right) \backslash B\left(x_{0}, 3 r / 2\right)}\left|\nabla_{x} p_{t}(x, y)\right| d x \\
& \lesssim t r^{n / 2-1}\left(\int_{B\left(x_{0}, 2 r\right) \backslash B\left(x_{0}, 3 r / 2\right)}\left|\nabla_{x} p_{t}(x, y)\right|^{2} d x\right)^{1 / 2}
\end{aligned}
$$

Arguing similarly to [16, Lemma 3] we find that there exists $\alpha>0$ so that

$$
\left(\int_{\Omega}\left|\nabla_{x} p_{t}(x, y)\right|^{2} e^{\frac{|x-y|^{2}}{\alpha t}} d x\right)^{1 / 2} \lesssim \frac{1}{t^{1 / 2+n / 4}}
$$

This implies that, for $y \in B\left(x_{0}, r\right)$,

$$
\left(\int_{B\left(x_{0}, 2 r\right) \backslash B\left(x_{0}, 3 r / 2\right)}\left|\nabla_{x} p_{t}(x, y)\right|^{2} d x\right)^{1 / 2} \lesssim \frac{e^{-\frac{r^{2}}{c t}}}{t^{1 / 2+n / 4}} .
$$

Inserting this into (35) we obtain the right hand side of (34) for $I_{1}$. This completes our proof. 
Lemma 4.7. If $f$ is a function supported in a ball $B$ with $4 B \cap \partial \Omega \neq \emptyset$ and $\|f\|_{L^{\infty}} \lesssim|B|^{-1 / p}$, then we have $\|f\|_{H_{M i}^{p}(\Omega)} \lesssim 1$.

Proof. We consider the family of balls $\{B(x, \delta(x) / 6): x \in B\}$ which covers the ball $B$. By Vitali's covering lemma we can pick a subfamily of balls denoted by $\left\{B_{j}:=B\left(x_{j}, \delta\left(x_{j}\right) / 2\right): j \in \mathbb{N}\right\}$ so that $B \subset \cup_{j \in \mathbb{N}} B\left(x_{j}, \delta\left(x_{j}\right) / 2\right)$ and the family $\left\{\frac{1}{3} B_{j}: j \in \mathbb{N}\right\}$ are pairwise disjoint. We now write

$$
f=\sum_{j} \frac{f \chi_{B_{j}}}{\sum_{i} \chi_{B_{i}}}=\sum_{j} \lambda_{j} A_{j}
$$

where

$$
\lambda_{j}=\left(\frac{\left|B_{j}\right|}{|B|}\right)^{1 / p} \quad \text { and } \quad A_{j}=\left(\frac{\left|B_{j}\right|}{|B|}\right)^{-1 / p} \frac{f \chi_{B_{j}}}{\sum_{i} \chi_{B_{i}}} .
$$

It is clear that $A_{j}$ is an $H_{M i}^{p}(\Omega)$-atom for every $j$. Indeed note that $\operatorname{supp} A_{j} \subset B_{j}$; moreover, we have

$$
\left\|A_{j}\right\|_{L^{\infty}} \leq\left(\frac{\left|B_{j}\right|}{|B|}\right)^{-1 / p}\|f\|_{L^{\infty}} \leq\left(\frac{\left|B_{j}\right|}{|B|}\right)^{-1 / p}|B|^{-1 / p}=\left|B_{j}\right|^{-1 / p} .
$$

Now since for each $j$ the ball $\frac{1}{3} B_{j}$ is contained in $4 B$ then

$$
\sum_{j}\left|\lambda_{j}\right|^{p}=\sum_{j} \frac{\left|B_{j}\right|}{|B|} \lesssim \sum_{j} \frac{\left|\frac{1}{3} B_{j}\right|}{|B|} \lesssim 1,
$$

and this gives $\|f\|_{H_{M i}^{p}(\Omega)} \lesssim 1$.

We now turn to the proof of Theorem 4.4.

Proof of Theorem 4.4: We shall only give the proof for the case $|\Omega|<\infty$. The remaining case $|\Omega|=\infty$ can be done in a similar way.

Since Theorem 1.4 applies to $L_{D}$ we may write $H_{L_{D}}^{p}$ for any of $H_{L_{D} \text {, max }}^{p}$ or $H_{L_{D} \text {,rad }}^{p}$. The inclusion $H_{M i}^{p}(\Omega) \hookrightarrow H_{L_{D}}^{p}(\Omega)$ is standard and a similar proof can be found in [15, Proposition 5.3]. Thus we will only demonstrate $H_{L_{D}}^{p}(\Omega) \hookrightarrow H_{M i}^{p}(\Omega)$ and to do this we draw upon the atomic characterization in Theorem 1.4. It suffices therefore to prove that for each $(p, M)_{L_{D}}$-atom $a$ with $M>\frac{n}{2}\left(\frac{1}{p}-1\right)$ we have

$$
\|a\|_{H_{M i}^{p}(\Omega)} \lesssim 1
$$

If $a$ is a $(p, M)_{L_{D}}$-atom of type (a) or (b) from Definition 1.1 associated to a ball $B$, and that ball satisfies $4 B \cap \partial \Omega \neq \emptyset$, then (36) holds by Lemma 4.7. It remains to consider the case $4 B \subset \Omega$. In this case, we have $a=L_{D}^{M} b$. We now write

$$
a=L_{D} e^{-r_{B}^{2} L_{D}} \tilde{b}+L_{D}\left(I-e^{-r_{B}^{2} L_{D}}\right) \tilde{b}=L_{D} e^{-r_{B}^{2} L_{D}} \tilde{b}+\left(I-e^{-r_{B}^{2} L_{D}}\right) a=: a_{1}+a_{2}
$$

where $\tilde{b}=L_{D}^{M-1} b$.

We only treat $a_{2}$ since $a_{1}$ can be handled similarly and is easier. To do this let $k_{0}$ be the positive integer such that $2^{k_{0}-1} r_{B} \leq \delta\left(x_{B}\right)<2^{k_{0}} r_{B}$. Then $k_{0} \geq 3$ necessarily. We set $S_{j}(B):=\left[2^{j+1} B \backslash 2^{j} B\right] \cap \Omega$ if $j>0$ and $S_{0}(B):=2 B$. We decompose $a_{2}$ as follows:

$$
\begin{aligned}
a_{2} & =\sum_{j=k_{0}-3}^{\infty} a_{2} \chi_{S_{j}(B)}+\sum_{j=0}^{k_{0}-3}\left(a_{2} \chi_{S_{j}(B)}-\frac{\chi_{S_{j}(B)}}{\left|S_{j}(B)\right|} \int_{S_{j}(B)} a_{2}\right)+\sum_{j=0}^{k_{0}-3} \frac{\chi_{S_{j}(B)}}{\left|S_{j}(B)\right|} \int_{S_{j}(B)} a_{2} \\
& =: \sum_{j=k_{0}-3}^{\infty} 2^{-j} \pi_{1, j}+\sum_{j=0}^{k_{0}-3} 2^{-j} \pi_{2, j}+\sum_{j=0}^{k_{0}-3} \frac{\chi_{S_{j}(B)}}{\left|S_{j}(B)\right|} \int_{S_{j}(B)} a_{2} .
\end{aligned}
$$

For the first summation it is clear that supp $\pi_{1, j} \subset S_{j}(B) \subset B_{j}:=2^{j+1} B$ and $4 B_{j} \cap \partial \Omega \neq \emptyset$ for all $j \geq k_{0}-3$. Moreover, we have

$$
\pi_{1, j}=2^{j}\left(I-e^{-r_{B}^{2} L_{D}}\right) a \cdot \chi_{S_{j}(B)} .
$$


For $j=0,1,2$ using the $L^{\infty}$-boundedness of $\left(I-e^{-r_{B}^{2} L_{D}}\right)$ we have

$$
\left\|\pi_{1, j}\right\|_{L^{\infty}} \lesssim\|a\|_{L^{\infty}} \lesssim|B|^{-1 / p} \sim\left|B_{j}\right|^{-1 / p} .
$$

For $j \geq 3$ we use

$$
\pi_{1, j}=2^{j} \int_{0}^{r_{B}^{2}} s L_{D} e^{-s L_{D}} a \cdot \chi_{S_{j}(B)} \frac{d s}{s} .
$$

and Gaussian bounds on the kernel of $s L_{D} e^{-s L_{D}}$ (which carry over from (D1)) to obtain

$$
\begin{aligned}
\left\|\pi_{1, j}\right\|_{L^{\infty}} & \leq 2^{j} \int_{0}^{r_{B}^{2}}\left\|s L_{D} e^{-s L_{D}} a\right\|_{L^{\infty}\left(S_{j}(B)\right)} \frac{d s}{s} \\
& \lesssim 2^{j} \int_{0}^{r_{B}^{2}} e^{-\frac{2^{j} r_{B}^{2}}{c s}}\|a\|_{L^{\infty}(B)} \frac{d s}{s} \\
& \lesssim\left|2^{j} B\right|^{-1 / p}=\left|B_{j}\right|^{-1 / p} .
\end{aligned}
$$

From (37), (38) and Lemma 4.7 we have $\left\|\pi_{1, j}\right\|_{H_{M i}^{p}(\Omega)} \lesssim 1$, and hence $\sum_{j=k_{0}-3}^{\infty} 2^{-j} \pi_{1, j} \in H_{M i}^{p}(\Omega)$.

For the terms $\pi_{2, j}$, we note that $\int \pi_{2, j}=0$ and $\operatorname{supp} \pi_{2, j} \subset B_{j}:=2^{j+1} B$ with $4 B_{j} \subset \Omega$. Arguing similarly to the estimates of $\pi_{1, j}$ we also find that $\left\|\pi_{2, j}\right\|_{L^{\infty}} \lesssim\left|B_{j}\right|^{-1 / p}$. Hence, $\pi_{2, j}$ is an $H_{M i}^{p}(\Omega)$-atom for each $j$. This implies $\sum_{j=k_{0}-3}^{\infty} 2^{-j} \pi_{2, j} \in H_{M i}^{p}(\Omega)$.

For the last term, we decompose further as follows:

$$
\sum_{j=0}^{k_{0}-3} \frac{\chi_{S_{j}(B)}}{\left|S_{j}(B)\right|} \int_{S_{j}(B)} a_{2}=\sum_{j=0}^{k_{0}-3}\left(\frac{\chi_{S_{j}(B)}}{\left|S_{j}(B)\right|}-\frac{\chi_{S_{j-1}(B)}}{\left|S_{j-1}(B)\right|}\right) \int_{2^{k_{0}-3} \backslash 2^{j} B} a_{2}+\frac{\chi_{2 B}}{|2 B|} \int_{2^{k_{0}-3} B} a_{2} .
$$

Now arguing as above, we can show that for $j=0,1, \ldots, k_{0}-3$

$$
\left\|\left(\frac{\chi_{S_{j}(B)}}{\left|S_{j}(B)\right|}-\frac{\chi_{S_{j-1}(B)}}{\left|S_{j-1}(B)\right|}\right) \int_{2^{k_{0}-3} \backslash 2^{j} B} a_{2}\right\|_{H_{M i}^{p}(\Omega)} \lesssim 2^{-j}
$$

For the remaining term $\frac{\chi_{2 B}}{|2 B|} \int_{2^{k_{0}-3} B} a_{2}$ we have

$$
\int_{2^{k_{0}-3} B} a_{2}=\int_{2^{k_{0}-3} B} \int_{0}^{r_{B}^{2}} s L_{D} e^{-s L_{D}} a(x) \frac{d s}{s} d x=\int_{0}^{r_{B}^{2}} \int_{B} \int_{2^{k_{0}-3} B} q_{s}(x, y) a(y) d x d y \frac{d s}{s} .
$$

On the other hand, by Lemma 4.6 we obtain

$$
\left|\int_{2^{k_{0}-3} B} q_{s}(x, y) a(y) d x\right| \lesssim \frac{\sqrt{s}}{2^{k_{0}} r_{B}} e^{-\frac{\left(2^{k_{0} r_{B}}\right)^{2}}{c s}} .
$$

Hence

$$
\int_{2^{k_{0}-3} B} a_{2} \lesssim \int_{0}^{r_{B}^{2}}\|a\|_{L^{1}} \frac{\sqrt{s}}{2^{k_{0}} r_{B}} e^{-\frac{\left(2^{k_{0} r_{B}}\right)^{2}}{c s}} \frac{d s}{s} \lesssim 2^{-k_{0}} e^{-c 2^{2 k_{0}}}|B|^{1-1 / p} \lesssim\left|B \| 2^{k_{0}-1} B\right|^{-1 / p},
$$

which implies

$$
\frac{\chi_{2 B}}{|2 B|} \int_{2^{k_{0}-3} B} a_{2} \lesssim\left|2^{k_{0}-2} B\right|^{-1 / p}
$$

As a consequence, $\frac{\chi_{2 B}}{|2 B|} \int_{2^{k_{0}-3} B} a_{2}$ is an $H_{M i}^{p}(\Omega)$-atom associated to the ball $2^{k_{0}-2} B$. It follows that

$$
\left\|\frac{\chi_{2 B}}{|2 B|} \int_{2^{k_{0}-3} B} a_{2}\right\|_{H_{M i}^{p}(\Omega)} \lesssim 1
$$

This completes our proof. 
4.3. Schrödinger operators with Dirichlet boundary conditions. Let $\Omega$ is a strongly Lipschitz domain of $\mathbb{R}^{n}$ with $n \geq 3$. This means that $\Omega$ is a proper open connected set in $\mathbb{R}^{n}$ and whose boundary is a finite union of parts of rotated graphs of Lipschitz maps, with at most one of these parts possibly infinite. The class of strongly Lipschitz domains includes special Lipschitz domains, bounded Lipschitz domains and exterior domains. See for example [3].

Let $0 \leq V \in R H_{\tilde{q}}\left(\mathbb{R}^{n}\right)$ with $\tilde{q}>n / 2$, i.e.,

$$
\left(\frac{1}{|B|} \int_{B} V(x)^{\tilde{q}} d x\right)^{1 / \tilde{q}} \leq C \frac{1}{|B|} \int_{B} V(x) d x
$$

for all balls $B \subset \mathbb{R}^{n}$.

We define

$$
W_{V, 0}^{1,2}(\Omega)=\left\{u \in W_{0}^{1,2}(\Omega): \int_{\Omega}|u(x)|^{2} V(x) d x<\infty\right\} .
$$

The Schrödinger $L$ on $\Omega$ with Dirichlet Boundary Condition (DBC) can be defined via the following sesquilinear form $\mathcal{Q}$ by setting

$$
\mathcal{Q}(f, g)=\int_{\Omega} \nabla f(x) \overline{\nabla g(x)} d x+\int_{\Omega} f(x) \overline{g(x)} V(x) d x .
$$

Then $L$ can be written as $L f=-\Delta f+V f$ where $f \in D(L)$ with

$$
D(L)=\left\{f \in W_{V, 0}^{1,2}(\Omega): \exists g \in L^{2}(\Omega): \mathcal{Q}(f, \phi)=\int_{\Omega} g(x) \overline{\phi(x)}, \forall \phi \in W_{V, 0}^{1,2}(\Omega)\right\} .
$$

For $V \in R H_{q}, q>n / 2$, we define the critical function $\rho(x)$ as follows:

$$
\rho(x)=\left\{r \in(0, \infty): \frac{1}{r^{n-2}} \int_{B\left(x_{0}, r\right)} V(y) d y \leq 1\right\} .
$$

Then there exist positive constants $C$ and $k_{0}$ so that

$$
\rho(y) \leq C \rho(x)\left(1+\frac{d(x, y)}{\rho(x)}\right)^{\frac{k_{0}}{k_{0}+1}}
$$

for all $x, y \in X$. See for example [31].

The critical function $\rho$ plays an important role in the rest of this section. Firstly it contributes to better bounds on the heat kernel for $L$ compared to those in (A2) and (A3).

Lemma $4.8([10])$. Let $L$ be a Schrödinger with $D B C$ on the strongly Lipschitz domain with $V \in R H_{\tilde{q}}, \tilde{q}>n / 2$. Then we have

(i) for any $N>0$ there exists $C=C(N)>0$ and $c>0$ so that for all $t>0$ and $x, y \in \Omega$,

$$
0 \leq p_{t}(x, y) \leq \frac{C}{t^{n / 2}} \exp \left(-\frac{|x-y|^{2}}{c t}\right)\left[1+\frac{\sqrt{t}}{\rho(x)}+\frac{\sqrt{t}}{\rho(y)}\right]^{-N} ;
$$

(ii) for any $N>0$ and $0<\delta<\min \left\{\gamma_{0}, 2-n / \tilde{q}\right\}$, there exists $C=C(N, \delta)>0$ and $c>0$ so that for all $t>0$ and $x, y, y^{\prime} \in \Omega$ with $\left|y-y^{\prime}\right|<\sqrt{t}$ and $0<t<\operatorname{diam} \Omega$,

$$
\left|p_{t}(x, y)-p_{t}\left(x, y^{\prime}\right)\right| \leq \frac{C}{t^{n / 2}}\left(\frac{\left|y-y^{\prime}\right|}{\sqrt{t}}\right)^{\delta} \exp \left(-\frac{|x-y|^{2}}{c t}\right)\left[1+\frac{\sqrt{t}}{\rho(x)}+\frac{\sqrt{t}}{\rho(y)}\right]^{-N} ;
$$

(iii) there exist $\alpha>0$ and $C=C(\alpha)$ so that for all $t>0$ and $y \in \Omega$,

$$
\left(\int_{\Omega}\left|\nabla_{x} p_{t}(x, y)\right| e^{\frac{|x-y|^{2}}{\alpha t}} d x\right)^{1 / 2} \leq \frac{C}{t^{1 / 2+n / 4}} .
$$

The function $\rho$ also gives us a useful covering of $\mathbb{R}^{n}$.

Lemma 4.9 ([18]). There exists a family of balls $\left\{B_{\alpha}\right\}_{\alpha \in \mathcal{I}}$ given by $B_{\alpha}=B\left(x_{\alpha}, \rho\left(x_{\alpha}\right)\right)$ satisfies

(i) $\bigcup_{\alpha \in \mathcal{I}} B\left(x_{\alpha}, \rho\left(x_{\alpha}\right)\right)=\mathbb{R}^{n}$; 
(ii) For every $\lambda \geq 1$ there exist constants $C$ and $N_{1}$ such that $\sum_{\alpha \in \mathcal{I}} \chi_{B\left(x_{\alpha}, \lambda \rho\left(x_{\alpha}\right)\right)} \leq C \lambda^{N_{1}}$.

Finally the function $\rho$ can be used to define an atomic Hardy space for $L$ which we now present.

Definition 4.10 (Hardy spaces for the DBC Schrödinger operator). Let $p \in\left(\frac{n}{n+1}, 1\right]$. A bounded, measurable function $a: \Omega \rightarrow \mathbb{R}$ supported in a ball $B$ is called an $(p, \rho)$-atom if either

(a) a is an $H_{M i}^{p}$-atom and $r_{B}<\rho\left(x_{B}\right) / 4$; or,

(b) $\|a\|_{L^{\infty}(\Omega)} \leq|B|^{-1 / p}, a \equiv 0$ on $\Omega \backslash B$ and $r_{B} \geq \rho\left(x_{B}\right) / 4$.

We now define the Hardy space $H_{\rho}^{p}(\Omega)$ as a set of all $f$ such that

$$
f=\sum_{j} \lambda_{j} a_{j}
$$

where $a_{j}$ are $(p, \rho)$-atoms and $\lambda_{j}$ are scalars such that $\sum_{j}\left|\lambda_{j}\right|^{p}<\infty$. We also set

$$
\|f\|_{H_{\rho}^{p}(\Omega)}^{p}=\inf \left\{\sum_{j}\left|\lambda_{j}\right|^{p}: f=\sum_{j} \lambda_{j} a_{j}\right\}
$$

where the infimum is taken over all such decompositions.

Theorem 4.11. Let $p \in\left(\frac{n}{n+\delta}, 1\right]$ where $\delta=\min \left\{\gamma_{0}, 2-n / \tilde{q}\right\}$. We have

$$
H_{L, \text { max }}^{p}(\Omega) \equiv H_{L, \text { rad }}^{p}(\Omega) \equiv H_{\rho}^{p}(\Omega), \quad \frac{n}{n+\delta}<p \leq 1 .
$$

Remark 4.12. We have the following remarks.

(a) It is important to note that due to Lemma 4.9 we may assume that each $(p, \rho)$-atom satisfying Definition 4.10 (b) also satisfies $\rho\left(x_{B}\right) / 4<r_{B} \leq \rho\left(x_{B}\right)$.

(b) Just like the Hardy spaces of Miyachi $H_{M i}^{p}(\Omega)$, the new Hardy space $H_{\rho}^{p}(\Omega)$ bears a close relationship with the following Hardy space of restriction related to critical function $\rho$. For $p \in\left(\frac{n}{n+1}, 1\right]$ we say a bounded function $a$ supported in a ball $B \subset \mathbb{R}^{n}$ is called an $(p, \rho)_{\mathbb{R}^{n-}}$ atom if either

(a) $\|a\|_{L^{\infty}(\Omega)} \leq|B|^{-1 / p}$,

(b) $\int a(x) d x=0$ if $r_{B}<\rho\left(x_{B}\right) / 4$.

We define

$$
\|f\|_{H_{\rho}^{p}(\Omega)}^{p}:=\inf \left\{\sum_{j}\left|\lambda_{j}\right|^{p}: f=\sum_{j} \lambda_{j} a_{j}\right\}
$$

where the infimum is taken over all such decompositions $f=\sum_{j} \lambda_{j} a_{j}$ with $(p, \rho)_{\mathbb{R}^{n} \text {-atoms }}$ $a_{j}$ and numbers $\lambda_{j}$ satisfying $\sum_{j}\left|\lambda_{j}\right|^{p}<\infty$. Then the Hardy space $H_{\rho}^{p}\left(\mathbb{R}^{n}\right)$ is defined as the completion in the quasi-norm $\|f\|_{H_{\rho}^{p}(\Omega)}^{p}$ of the set $\left\{f \in L^{2}: f=\sum_{j} \lambda_{j} a_{j}\right\}$.

The Hardy space of restriction related to $\rho$ is now defined as

$$
H_{\rho, r}^{p}(\Omega)=\left\{f: \text { there exists } F \in H_{\rho}^{p}\left(\mathbb{R}^{n}\right) \text { so that }\left.F\right|_{\Omega}=f\right\}
$$

with the norm

$$
\|f\|_{H_{\rho, r}^{p}(\Omega)}=\inf \left\{\|F\|_{H_{\rho}^{p}\left(\mathbb{R}^{n}\right)}: F \in H_{\rho}^{p}\left(\mathbb{R}^{n}\right),\left.F\right|_{\Omega}=f\right\} .
$$

Then it was proved in [10] that if either $\Omega$ is bounded or $\Omega^{c}$ is unbounded, then $H_{\rho, r}^{p}(\Omega)=$ $H_{\rho}^{p}(\Omega)$ for all $\frac{n}{n+\delta}<p \leq 1$ with $\delta=\min \left\{\gamma_{0}, 2-n / \tilde{q}\right\}$. This and Theorem 4.11 immediately imply the following result:

Corollary 4.13. Let $\Omega$ be a strongly Lipschitz domain such that either $\Omega$ is bounded or $\Omega^{c}$ is unbounded. Let $p \in\left(\frac{n}{n+\delta}, 1\right]$ where $\delta=\min \left\{\gamma_{0}, 2-n / \tilde{q}\right\}$. Then we have

$$
H_{L, \text { max }}^{p}(\Omega) \equiv H_{L, \text { rad }}^{p}(\Omega) \equiv H_{\rho, r}^{p}(\Omega), \quad \frac{n}{n+\delta}<p \leq 1 .
$$

As in the proof of Theorem 4.11 we require certain kernel estimates first. 
Lemma 4.14. Let $q_{t}(x, y)$ be the kernel of $t L e^{-t L}$. Suppose that $x_{0} \in \Omega$ and $0<r<\rho\left(x_{0}\right) / 4$ so that $B\left(x_{0}, 2 r\right) \subset \Omega$. Then we have

$$
\left|\int_{B\left(x_{0}, 2 r\right)} q_{t}(x, y) d x\right| \lesssim\left(\frac{\sqrt{t}}{r}\right)^{2-n / q}
$$

for all $0<t<\rho\left(x_{0}\right)^{2}$ and all $y \in B\left(x_{0}, r\right)$.

Proof. As in the proof of Lemma 4.6, we take $\psi \in C_{c}^{\infty}\left(x_{0}, 2 r\right)$ so that $\psi=1$ on $B\left(x_{0}, 3 r / 2\right)$ and $|\nabla \psi| \lesssim 1 / r$. Then we have

$$
\int_{B\left(x_{0}, 2 r\right)} q_{t}(x, y) d x=\int_{B\left(x_{0}, 2 r\right)} q_{t}(x, y) \psi(x) d x+\int_{B\left(x_{0}, 2 r\right)} q_{t}(x, y)[1-\psi(x)] d x=: J_{1}+J_{2} .
$$

We can argue similarly to $I_{2}$ of Lemma 4.6 to get $\left|I_{2}\right| \lesssim e^{-r^{2} / c t}$.

To estimate $I_{1}$ we use (39) and (40) to deduce

$$
J_{1}=t \int_{\Omega} \nabla_{x} p_{t}(x, y) \cdot \nabla \psi(x) d x+t \int_{\Omega} p_{t}(x, y) V(x) \psi(x) d x=: J_{11}+J_{12} .
$$

Then again arguing as in the proof of estimate $I_{1}$ from Lemma 4.6 we can obtain $\left|J_{11}\right| \lesssim e^{-r^{2} / c t}$.

Using [20, Lemma 5.1] we conclude that

$$
\left|J_{12}\right| \lesssim\left(\frac{\sqrt{t}}{\rho(y)}\right)^{2-n / \tilde{q}} .
$$

On the other hand, since $\left|y-x_{0}\right|<r<\rho\left(x_{0}\right) / 4$, from (42) we have $\rho(y) \sim \rho\left(x_{0}\right)>4 r$. Hence,

$$
\left|J_{12}\right| \lesssim\left(\frac{\sqrt{t}}{r}\right)^{2-n / \tilde{q}}
$$

Collecting all estimates $I_{2}, J_{11}$ and $J_{12}$ we get the desired estimate (45).

We are now ready to give the proof of Theorem 4.11.

Proof of Theorem 4.11: We shall only give the proof for the case $|\Omega|<\infty$ since the remaining case $|\Omega|=\infty$ can be done similarly.

We will first show that $H_{\rho}^{p}(\Omega) \subset H_{L, \text { rad }}^{p}(\Omega)$. Indeed, let $a$ be a $(p, \rho)$-atom associated to a ball $B$. Now if $r_{B}<\rho\left(x_{B}\right) / 4$, then in this case $a$ is a $H_{M i}^{p}(\Omega)$-atom and a standard argument shows that

$$
\left\|a_{L}^{+}\right\|_{L^{p}(\Omega)} \lesssim 1
$$

On the other hand if $\rho\left(x_{B}\right) / 4<r_{B} \leq \rho\left(x_{B}\right)$, then we split

$$
\left\|a_{L}^{+}\right\|_{L^{p}}^{p} \leq\left\|a_{L}^{+}\right\|_{L^{p}(4 B)}^{p}+\left\|a_{L}^{+}\right\|_{L^{p}(\Omega \backslash 4 B)}^{p}:=I_{1}+I_{2} .
$$

Hölder's inequality and the estimate $\left\|a_{L}^{+}\right\|_{L^{\infty}} \lesssim\|a\|_{L^{\infty}}$ allow us to readily conclude that $I_{1} \lesssim 1$.

We turn to the second term $I_{2}$. Note firstly that $r_{B} \sim \rho\left(x_{0}\right) \sim \rho(y)$, and secondly that $|x-y| \sim\left|x-x_{0}\right|$ holds whenever $y, x_{0} \in B$ and $x \in(4 B)^{c}$. These facts in tandem with (44) allow us to obtain, for $N>n(1-p) / p$,

$$
\begin{aligned}
I_{2} & \lesssim \int_{(4 B)^{c}} \sup _{t>0}\left[\int_{B} \frac{1}{t^{n / 2}} \exp \left(-\frac{|x-y|^{2}}{c t}\right)\left(\frac{\rho(y)}{\sqrt{t}}\right)^{N}|a(y)| d y\right]^{p} d x \\
& \lesssim \int_{(4 B)^{c}} \sup _{t>0}\left[\int_{B} \frac{1}{t^{n / 2}} \exp \left(-\frac{\left|x-x_{0}\right|^{2}}{c t}\right)\left(\frac{r_{B}}{\sqrt{t}}\right)^{N}|a(y)| d y\right]^{p} d \mu(x) \\
& \lesssim \int_{(4 B)^{c}}\left[\int_{B} \frac{1}{\left|x-x_{0}\right|^{n}}\left(\frac{r_{B}}{\left|x-x_{0}\right|}\right)^{N}|a(y)| d y\right]^{p} d x \\
& \lesssim 1 .
\end{aligned}
$$

This completes the direction $H_{\rho}^{p}(\Omega) \subset H_{L, \text { rad }}^{p}(\Omega)$. The reverse direction can be done in a similar way to that of Theorem 4.4 and will be omitted. 
4.4. Fourier-Bessel operators on $((0,1), d x)$. For $\nu>-1$, we consider the following differential operator

$$
L=-\frac{d^{2}}{d x^{2}}+\frac{\nu^{2}-1 / 4}{x^{2}} .
$$

Let $\left\{\lambda_{k, \nu}\right\}_{k \geq 1}$ denote the sequence of successive positive zeros of the Bessel function $J_{\nu}$ and consider

$$
\psi_{k}^{\nu}(x)=d_{k, \nu} \lambda_{k, \nu}^{1 / 2} J_{\nu}\left(\lambda_{k, \nu} x\right) x^{1 / 2}
$$

where $x \in(0,1)$ and $d_{k, \nu}=\sqrt{2}\left|\lambda_{k, \nu} J_{\nu+1}\left(\lambda_{k, \nu}\right)\right|^{-1}$.

Then the system $\left\{\psi_{k}^{\nu}\right\}_{k}$ forms an orthornomal basis for $((0,1), d x)$. It is well-known that

$$
L \psi_{k}^{\nu}(x)=\lambda_{k, \nu}^{2} \psi_{k}^{\nu}(x) .
$$

The operator $L$ has a non-negative self-adjoint extension which is still denoted by $L$ with domain

$$
D(L)=\left\{f \in L^{2}((0,1), d x): \sum_{k=1}^{\infty} \lambda_{k, \nu}^{4}\left|\left\langle f, \psi_{k}^{\nu}\right\rangle\right|^{2}<\infty\right\} .
$$

This operator is called the Bessel operator on $((0,1), d x)$.

In order to consider the maximal function characterization for the Hardy spaces associated to $L$, as in [4] we consider the intervals:

$$
\mathcal{J}_{j}= \begin{cases}\left(1-2^{-j}, 1-2^{-j-1}\right], & j \geq 1 \\ \left(2^{j-1}, 2^{j}\right], & j \leq-1 .\end{cases}
$$

which are depicted in Figure 1.

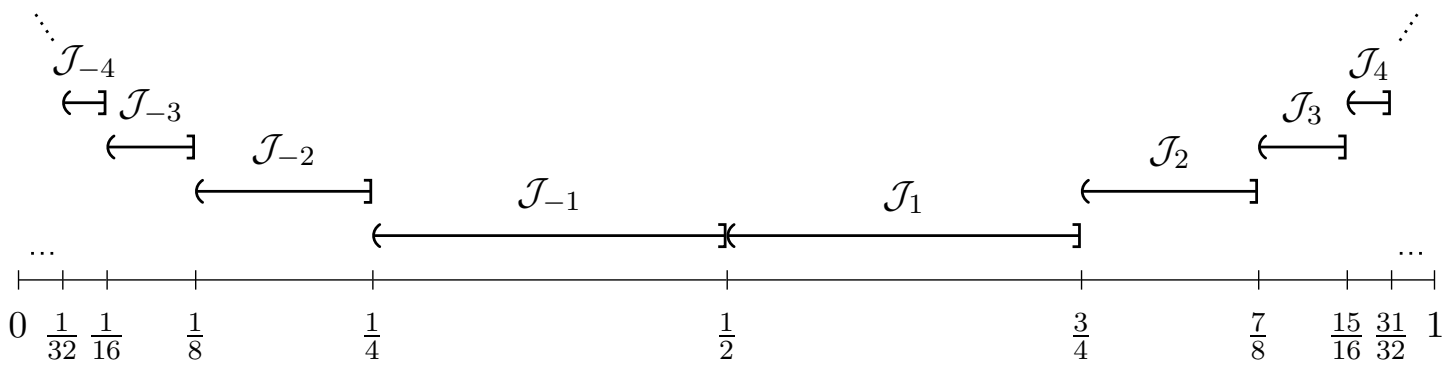

FiguRE 1. Intervals $\mathcal{J}_{j}$

It is obvious that the family $\{\mathcal{J}\}_{j \in \mathbb{N}}$ is pairwise disjoint and $(0,1)=\bigcup_{j \in \mathbb{N}} \mathcal{J}_{j}$. For each $j \in \mathbb{Z}^{*}$ we also denote $\mathcal{J}_{j}^{*}=\frac{1}{10} \mathcal{J}_{j}$. We now consider the following atoms.

Definition 4.15. Let $\frac{1}{2}<p \leq 1$. A function a is a type (a) atom associated with an interval $I \subseteq(0,1)$ if

(i) $\operatorname{supp} a \subset I$

(ii) $\|a\|_{L^{\infty}} \leq|I|^{-1 / p}$

(iii) $\int a(x) d x=0$

A function $a$ is a type (b) atom if

$$
a(x)=\frac{\chi_{\mathcal{J}_{j}}(x)}{\left|\mathcal{J}_{j}\right|^{1 / p}}
$$

for some $j \in \mathbb{N}$. We say a function a is an $H^{p}((0,1), d x)$-atom if it is either a type (a) or type (b) atom. 
Definition 4.16 (Atomic Hardy spaces on $((0,1), d x))$. Given $p \in\left(\frac{1}{2}, 1\right], q \in[1, \infty] \cap(p, \infty]$, we say that $f=\sum \lambda_{j} a_{j}$ is an atomic $H^{p}((0,1), d x)$-representation if $\left\{\lambda_{j}\right\}_{j=0}^{\infty} \in l^{p}$, each $a_{j}$ is an $H^{p}((0,1), d x)$-atom, and the sum converges in $L^{2}(X)$. The space $H_{a t}^{p}((0,1), d x)$ is then defined as the completion of

$$
\left\{f \in L^{2}: f \text { has an atomic } H^{p}((0,1), d x) \text {-representation }\right\},
$$

with the norm given by

$\|f\|_{H_{a t}^{p}((0,1), d x)}=\inf \left\{\left(\sum\left|\lambda_{j}\right|^{p}\right)^{1 / p}: f=\sum \lambda_{j} a_{j}\right.$ is an atomic $H^{p}((0,1), d x)$-representation $\}$.

As in Definition 1.3 we denote by $H_{L, \text { rad }}^{p}((0,1), d x)$ and $H_{L, \max }^{p}((0,1), d x)$ respectively the maximal Hardy spaces defined via radial and non-tangential maximal functions associated to $L$.

Our main result in this section is the following:

Theorem 4.17. Let $\nu>-1 / 2$. Let $p \in\left(\frac{1}{1+\delta}, 1\right]$ where $\delta=\min \{1, \nu+1 / 2\}$. Then we have

$$
H_{a t}^{p}((0,1), d x) \equiv H_{L, \mathrm{rad}}^{p}((0,1), d x) \equiv H_{L, \max }^{p}((0,1), d x)
$$

with equivalent norms.

Remark 4.18. Note that it was proved in [19] that

$$
H_{a t}^{1}((0,1), d x) \equiv H_{\sqrt{L}, \mathrm{rad}}^{1}((0,1), d x) \equiv H_{\sqrt{L}, \max }^{1}((0,1), d x) .
$$

Hence the results of Theorem 4.17 can be viewed as an extension of those in [19] to the range $p$ below 1 .

In order to give the proof of Theorem 4.17 we need the following technical material. Firstly we collect together some estimates on the kernels and their derivatives.

Lemma 4.19 ([28] in Theorem 1.1). For $\nu>-1$ we have

$$
p_{t}(x, y) \approx \frac{(x y)^{\nu+1 / 2}(1+t)^{\nu+2}}{(t+x y)^{\nu+1 / 2}}\left(1 \wedge \frac{(1-x)(1-y)}{t}\right) \frac{1}{\sqrt{t}} e^{-\frac{|x-y|^{2}}{4 t}-\lambda_{1, \nu}^{2} t}
$$

for $x, y \in X$ and $t>0$.

When $\nu>-1 / 2$, a simple calculation applied to the bounds in Lemma 4.19 gives

$$
\left|p_{t}(x, y)\right| \lesssim \frac{(x y)^{\nu+1 / 2}}{(t+x y)^{\nu+1 / 2}} \frac{1}{\sqrt{t}}\left(1 \wedge \frac{(1-x)(1-y)}{t}\right) e^{-\frac{|x-y|^{2}}{c t}}
$$

for all $x, y \in X$ and $t>0$. Note that when $-1<\nu<-1 / 2$ the Gaussian upper bounds for the kernel $p_{t}(x, y)$ may fail. Hence and for this reason we restrict ourself to the case $\nu>-1 / 2$.

Lemma 4.20. For $\nu>-1$ we have

$$
\left|\partial_{x} p_{t}(x, y)\right| \lesssim \frac{1}{t} e^{-(x-y)^{2} / 4 t}+\frac{1}{x} p_{t}(x, y) .
$$

for all $x, y \in X$ and $t>0$.

As a consequence, if $\nu>-1 / 2$, then we have

$$
\left|\partial_{x} p_{t}(x, y)\right| \lesssim \frac{1}{x t} e^{-(x-y)^{2} / 4 t}
$$

for all $x, y \in X$ and $t>0$.

Proof. We have

$$
\left|\partial_{x} p_{t}(x, y)\right| \lesssim\left|\left(\partial_{x}-\frac{\nu+1 / 2}{x}\right) p_{t}(x, y)\right|+\frac{|\nu+1 / 2|}{x} p_{t}(x, y) .
$$

Using the argument in [19, Lemma 2.4] we find that

$$
\left|\left(\partial_{x}-\frac{\nu+1 / 2}{x}\right) p_{t}(x, y)\right| \lesssim \frac{1}{t} e^{-(x-y)^{2} / 4 t} .
$$


This yields (49) as desired.

The estimate (50) follows from (49) and (48).

Let us define the notion of "intervals" in $(0,1)$. For $x \in(0,1)$ and $r>0$ we denote by

$$
I_{r}(x)=(x-r, x+r) \cap(0,1)
$$

the interval centred at $x$ of radius $r$. Henceforth and unless otherwise specified, by an interval $I$ in $(0,1)$ we shall mean $I=I_{r_{I}}\left(x_{I}\right)$ for some fixed centre $x_{I}$ and radius $r_{I}$.

We define the critical function for $L$ by

$$
\rho(x):=\frac{1}{3} \min \{x, 1-x\}, \quad x \in(0,1)
$$

For $x \in(0,1)$ and $\rho$ defined as in $(52)$, we denote $I_{\rho}(x)=I_{\rho(x)}(x)$. Such an interval is called a critical interval.

We have the following result whose easy proof we omit.

Lemma 4.21. If $I$ is an interval with $r_{I} \leq \rho\left(x_{I}\right)$ then we have, for all $x \in I$

(i) $x \sim x_{I}$;

(ii) $\rho(x) \sim \rho\left(x_{I}\right)$;

(iii) $r_{I} \lesssim x$.

Denote by $Q_{t}$ the operator

$$
Q_{t}:=t L e^{-t L}
$$

and $q_{t}(x, y)$ the kernel of $Q_{t}$. It is well-known that the Gaussian upper bound can be transfered to the kernel $q_{t}(x, y)$, i.e.,

$$
\left|q_{t}(x, y)\right| \lesssim \frac{1}{\sqrt{t}} e^{-\frac{|x-y|^{2}}{c t}}
$$

for all $x, y \in X$ and $t>0$. We apply (53) to obtain the following.

Lemma 4.22. For any interval $I$ with $r_{I} \leq \rho\left(x_{I}\right)$ we have

$$
\left|\int_{I} q_{t}(x, y) d x\right| \lesssim \frac{t}{r_{I}^{2}}
$$

for any $\forall y \in \frac{1}{2} I$ and $t>0$.

Proof. Define the cutoff function $\varphi \in C_{c}^{\infty}(X)$ supported in $I$ with $0 \leq \varphi \leq 1$, equal to 1 on $\frac{3}{4} I$ and whose derivative satisfies $\left|\varphi^{\prime}(x)\right| \lesssim 1 / r_{I}$. Then

$$
\left|\int_{I} q_{t}(x, y) d x\right| \lesssim\left|\int_{X} t \partial_{t} p_{t}(x, y) \varphi(x) d x\right|+\left|\int_{I \backslash \frac{3}{4} I} q_{t}(x, y)[1-\varphi(x)] d x\right|=: I_{1}+I_{2}
$$

For the term $I_{2}$, using (53) and that $|x-y| \sim r_{I}$ we have

$$
I_{2} \lesssim \int_{I \backslash \frac{3}{4} I} \frac{1}{\sqrt{t}} e^{-\frac{|x-y|^{2}}{4 t}} d x \lesssim e^{-\frac{r_{I}^{2}}{c t}} \int_{I \backslash \frac{3}{4} I} \frac{1}{\sqrt{t}} e^{-\frac{|x-y|^{2}}{8 t}} d x \lesssim e^{-\frac{r_{I}^{2}}{c t}}
$$

For the first term, since $\partial_{t} p_{t}(\cdot, y)=-L p_{t}(\cdot, y)$, then

$$
I_{1} \lesssim\left|\int_{X} t \partial_{x x}^{2} p_{t}(x, y) \varphi(x) d x\right|+\left|\nu^{2}-1 / 4\right|\left|\int_{\frac{3}{4} I} t p_{t}(x, y) \varphi(x) \frac{d x}{x^{2}}\right|=: I_{11}+I_{12} .
$$

Now (48) and (iii) in Lemma 4.21 implies that $I_{12} \lesssim t / r_{I}^{2}$.

Integration by parts gives

$$
I_{11}=\left|\int_{X} t \partial_{x} p_{t}(x, y) \partial_{x} \varphi(x) d x\right|
$$


and along with estimate (50), part (iii) of Lemma 4.21 and the fact that $\left|\varphi^{\prime}(x)\right| \lesssim r_{I}^{-1}$ yields

$$
I_{11} \lesssim \frac{t}{r_{I}}\left|\int_{\frac{3}{4} I} \frac{1}{x \sqrt{t}} e^{-\frac{|x-y|^{2}}{4 t}} d x\right| \sim \frac{t}{r_{I}}\left|\int_{\frac{3}{4} I} \frac{1}{r_{I} \sqrt{t}} e^{-\frac{|x-y|^{2}}{4 t}} d x\right| \lesssim \frac{t}{r_{I}^{2}}
$$

completing our proof.

We now turn to the action of the radial maximal operator on atoms. Note that the intervals $\mathcal{J}_{j}^{*}$ has been defined in the comments after (47).

Lemma 4.23. Let $\nu>-1 / 2$ and $\frac{1}{1+\delta}<p \leq 1$ with $\delta=\min \{1, \nu+1 / 2\}$. Suppose that $a$ is either

(i) a type (b) $H^{p}((0,1), d x)$-atom, or

(ii) a type (a) $H^{p}((0,1), d x)$-atom supported in $\mathcal{J}_{j}^{*}$ for some $j \in \mathbb{Z}^{*}$.

Then there exists $C>0$ independent of a so that

$$
\left\|\sup _{t>0}\left|e^{-t L} a\right|\right\|_{L^{p}((0,1), d x)} \leq C .
$$

Proof of Lemma 4.23. Proof of part (i). Since $a$ is an $H^{p}((0,1), d x)$-atom of type (b), then $a=\frac{\chi_{I}}{|I|^{1 / p}}$ where $I \equiv \mathcal{J}_{j}$ some $j \in \mathbb{Z} \backslash\{0\}$.

$$
\left\|\sup _{t>0}\left|e^{-t L} a\right|\right\|_{L^{p}((0,1), d x)} \lesssim\left\|\sup _{t>0}\left|e^{-t L} a\right|\right\|_{L^{p}(2 I)}+\left\|\sup _{t>0}\left|e^{-t L} a\right|\right\|_{L^{p}\left((2 I)^{c}\right)}=: E_{1}+E_{2} .
$$

It is easy to see that

$$
E_{1} \lesssim|2 I|^{1 / p}\left\|\sup _{t>0}\left|e^{-t L} a\right|\right\|_{L^{\infty}(2 I)} \lesssim|2 I|^{1 / p}\|a\|_{L^{\infty}(2 I)}=1 .
$$

We handle $E_{2}$ by studying the pointwise bounds on $\sup _{t>0}\left|e^{-t L} a(x)\right|$. Firstly by the heat kernel bounds (48), and that $|x-y| \sim\left|x-x_{I}\right|$ whenever $x \in(2 I)^{c}$, we have

$$
\begin{aligned}
\sup _{t>0}\left|e^{-t L} a(x)\right| \lesssim & \sup _{0<t<r_{I}^{2}}|I|^{-1 / p} \int_{I} \frac{1}{\sqrt{t}} e^{-\frac{|x-y|^{2}}{c t}} d y \\
& \quad+\sup _{t \geq r_{I}^{2}}|I|^{-1 / p} \int_{I} \frac{(x y)^{\nu+1 / 2}}{(t+x y)^{\nu+1 / 2}} \frac{1}{\sqrt{t}}\left(1 \wedge \frac{(1-x)(1-y)}{t}\right) e^{-\frac{\left|x-x_{I}\right|^{2}}{c t}} d y \\
= & : E_{21}(x)+E_{22}(x) .
\end{aligned}
$$

It is straightforward that

$$
E_{21}(x) \lesssim|I|^{1-1 / p} \frac{1}{\left|x-x_{I}\right|} \frac{r_{I}^{2}}{\left|x-x_{I}\right|^{2}}
$$

which implies $\left\|E_{21}\right\|_{L^{p}\left((2 I)^{c}\right)} \lesssim 1$ provided $p \in(1 / 2,1)$.

We divide the calculation for $E_{22}$ into two cases.

Case 1: $I \equiv \mathcal{J}_{j}, j>0$. In this case we have $(1-x) \lesssim\left|x-x_{I}\right|$ and $(1-y) \sim r_{I}$. Hence

$$
E_{22}(x) \lesssim \sup _{t \geq r_{I}^{2}}|I|^{-1 / p} \int_{I} \frac{1}{\sqrt{t}} \frac{\left|x-x_{I}\right| r_{I}}{t} e^{-\frac{\left|x-x_{I}\right|^{2}}{c t}} d y \lesssim|I|^{1-1 / p} \frac{1}{\left|x-x_{I}\right|} \frac{r_{I}}{\left|x-x_{I}\right|},
$$

which implies $\left\|E_{22}\right\|_{L^{p}\left((2 I)^{c}\right)} \lesssim 1$ whenever $p \in(1 / 2,1)$.

Case 2: $I \equiv \mathcal{J}_{j}, j<0$. In this case we have $x \lesssim\left|x-x_{I}\right|$ and $y \sim r_{I}$. Hence,

$$
\begin{aligned}
E_{22}(x) & \lesssim \sup _{t \geq r_{I}^{2}}|I|^{-1 / p} \int_{I}\left(\frac{x y}{t}\right)^{\nu+1 / 2} \frac{1}{\sqrt{t}} e^{-\frac{\left|x-x_{I}\right|^{2}}{c t}} d y \\
& \lesssim \sup _{t \geq r_{I}^{2}}|I|^{-1 / p} \int_{I}\left(\frac{r_{I}\left|x-x_{I}\right|}{t}\right)^{\nu+1 / 2} \frac{1}{\sqrt{t}} e^{-\frac{\left|x-x_{I}\right|^{2}}{c t}} d y d y \\
& \lesssim|I|^{1-1 / p} \frac{1}{\left|x-x_{I}\right|}\left(\frac{r_{I}}{\left|x-x_{I}\right|}\right)^{\nu+1 / 2}
\end{aligned}
$$


which yields $\left\|E_{22}\right\|_{L^{p}\left((2 I)^{c}\right)} \lesssim 1$, provided that $p \in\left(\frac{1}{1+\delta}, 1\right]$ with $\delta=\min \{1, \nu+1 / 2\}$.

Collecting together the estimates for $E_{21}$ and $E_{22}$ we obtain $E_{2} \lesssim 1$, completing the proof of part (i).

We now prove part (ii). Suppose that $a$ is an $H^{p}((0,1), d x)$-atom of type (a) associated to some interval $I \subset \mathcal{J}_{j}^{*}$.

We write

$$
\left\|\sup _{t>0}\left|e^{-t L} a\right|\right\|_{L^{p}((0,1), d x)} \lesssim\left\|\sup _{t>0}\left|e^{-t L} a\right|\right\|_{L^{p}(2 I)}+\left\|\sup _{t>0}\left|e^{-t L} a\right|\right\|_{L^{p}\left((2 I)^{c}\right)}=: F_{1}+F_{2} .
$$

By arguing similarly to $E_{1}$ in the proof of part (i) we have $F_{1} \lesssim 1$.

To handle $F_{2}$ we use the cancellation property of $a$ to write

$$
e^{-t L} a(x)=\int_{I}\left[p_{t}(x, y)-p_{t}\left(x, x_{I}\right)\right] a(y) d y .
$$

Then for $x \in(2 I)^{c}$ we may apply Lemma 4.20, the bounds (48), and the fact that $|x-y| \sim\left|x-x_{I}\right|$ whenever $y \in I$ to obtain

$$
\begin{aligned}
\sup _{t>0}\left|e^{-t L} a(x)\right| \lesssim \sup _{t>0} \int_{I} \frac{\left|y-x_{I}\right|}{\sqrt{t}} \frac{1}{\sqrt{t}} e^{-\frac{\left|x-x_{I}\right|^{2}}{c t}}|a(y)| d y & \\
& \quad+\sup _{t>0} \int_{I}\left(\frac{x y}{t+x y}\right)^{\nu+1 / 2} \frac{\left|y-x_{I}\right|}{y} \frac{1}{\sqrt{t}} e^{-\frac{\left|x-x_{I}\right|^{2}}{c t}}|a(y)| d y \\
= & : F_{21}(x)+F_{22}(x) .
\end{aligned}
$$

Since the variable $y$ belongs to $I$ it is then clear that

$$
F_{21}(x) \lesssim \sup _{t>0}\|a\|_{L^{\infty}} \int_{I} \frac{r_{I}}{\sqrt{t}} \frac{1}{\sqrt{t}} e^{-\frac{\left|x-x_{I}\right|^{2}}{c t}} d y \lesssim|I|^{1-1 / p} \frac{r_{I}}{\left|x-x_{I}\right|} \frac{1}{\left|x-x_{I}\right|}
$$

which implies $\left\|F_{21}\right\|_{L^{p}\left((2 I)^{c}\right)} \lesssim 1$.

For the expression $F_{22}$ we further subdivide

$$
F_{22}=F_{22} \chi_{3 \mathcal{J}_{j} \backslash 2 I}+F_{22} \chi_{\left(3 \mathcal{J}_{j}\right)^{c}} .
$$

Now whenever $x \in 3 \mathcal{J}_{j} \backslash 2 I$ we have the inequality $y^{-1} \lesssim\left|x-x_{I}\right|^{-1}$. Thus the first term can be controlled by

$$
F_{22}(x) \chi_{3 \mathcal{J}_{j} \backslash 2 I}(x) \lesssim \sup _{t>0} \int_{I} \frac{\left|y-x_{I}\right|}{\left|x-x_{I}\right|} \frac{1}{\sqrt{t}} e^{-\frac{\left|x-x_{I}\right|^{2}}{c t}}|a(y)| d y \lesssim|I|^{1-1 / p} \frac{r_{I}}{\left|x-x_{I}\right|} \frac{1}{\left|x-x_{I}\right|},
$$

which yields $\left\|F_{22}\right\|_{L^{p}\left(3 \mathcal{J}_{j} \backslash 2 I\right)} \lesssim 1$.

For the second term we consider two cases.

Case 1: $j>0$. In this situation $y \sim 1$, implying $y^{-1} \lesssim\left|x-x_{I}\right|^{-1}$ and therefore,

$$
F_{22}(x) \chi_{\left(3 \mathcal{J}_{j}\right)^{c}}(x) \lesssim \sup _{t>0} \int_{I} \frac{r_{I}}{\left|x-x_{I}\right|} \frac{1}{\sqrt{t}} e^{-\frac{\left|x-x_{I}\right|^{2}}{c t}}|a(y)| d y \lesssim|I|^{1-1 / p} \frac{r_{I}}{\left|x-x_{I}\right|} \frac{1}{\left|x-x_{I}\right|}
$$

Case 2: $j<0$. In this case $\left(3 \mathcal{J}_{j}\right)^{c}=\left(6 r_{\mathcal{J}_{j}}, 1\right)$ and hence $x \sim x-x_{I}$. Then we have

$$
\begin{aligned}
F_{22}(x) \chi_{\left(3 \mathcal{J}_{j}\right)^{c}}(x) & \lesssim \sup _{t>0} \int_{I}\left(\frac{x y}{t}\right)^{\delta} \frac{r_{I}}{y} \frac{1}{\sqrt{t}} e^{-\frac{\left|x-x_{I}\right|^{2}}{c t}}|a(y)| d y \\
& \lesssim \sup _{t>0} \int_{I}\left(\frac{y\left|x-x_{I}\right|}{t}\right)^{\delta}\left(\frac{r_{I}}{y}\right)^{\delta} \frac{1}{\left|x-x_{I}\right|} e^{-\frac{\left|x-x_{I}\right|^{2}}{2 c t}}|a(y)| d y \\
& \lesssim|I|^{1-1 / p}\left(\frac{r_{I}}{\left|x-x_{I}\right|}\right)^{\delta} \frac{1}{\left|x-x_{I}\right|}
\end{aligned}
$$

where $\delta=\min \{1, \nu+1 / 2\}$.

Taking into account the bounds in both cases we conclude $\left\|F_{22}\right\|_{L^{p}\left(\left(3 \mathcal{J}_{j}\right)^{c}\right)} \lesssim 1$.

On combining our estimates for $F_{21}$ and $F_{22}$ we then have $F_{2} \lesssim 1$, completing our proof of the Lemma. 
We are now ready to prove the main theorem of this section.

Proof of Theorem 4.17: We split the proof into two steps.

Step 1: $H_{L, \mathrm{rad}}^{p}((0,1), d x) \subset H_{a t}^{p}((0,1), d x)$.

Suppose that $a$ is a $(p, M)_{L^{-a t o m}}$ as in Definition 1.1 associated to an interval $I$. We consider two cases: $4 I \cap(0,1)^{c} \neq \emptyset$ and $4 I \subset(0,1)$.

Case 1: $4 I \cap(0,1)^{c} \neq \emptyset$. In this situation, it easy to see that if $x_{I} \in \mathcal{J}_{j}$ for some $j \in \mathbb{Z}$, then $|I| \sim\left|\mathcal{J}_{j}\right|$. Hence, using the decomposition

$$
a=\left[a-\frac{\chi_{\mathcal{I}_{j}}}{\left|\mathcal{I}_{j}\right|} \int a\right]+\frac{\chi_{\mathcal{I}_{j}}}{\left|\mathcal{I}_{j}\right|} \int a=: \tilde{a}_{1}+\tilde{a}_{2}
$$

We see that $a_{1}$ is an $H^{p}((0,1), d x)$ of type (a), while $a_{2}$ is an $H^{p}((0,1), d x)$ atom of type (b). Thus $a \in H_{a t}^{p}((0,1), d x)$.

Case 2: $4 I \subset(0,1)$. In this case, $a$ can be expressed in the form $a=L b$. We now write

$$
a=L e^{-r_{I}^{2} L} b+L\left(I-e^{-r_{I}^{2} L}\right) b=L e^{-r_{I}^{2} L} b+\left(I-e^{-r_{I}^{2} L}\right) a=a_{1}+a_{2}
$$

where $b$ is supported in $B$ and satisfies $\|b\|_{L^{\infty}} \leq r_{I}^{2}|I|^{-1 / p}$.

We take care $a_{2}$ only, since $a_{1}$ can be similarly treated. We choose $k_{0} \in \mathbb{N}$ so that $2^{k_{0}-1} r_{I} \leq$ $\frac{4}{3} \min \left\{x_{I}, 1-x_{I}\right\}=4 \rho\left(x_{I}\right)<2^{k_{0}} r_{I}$. Hence $k_{0} \geq 3$. We set $S_{j}(I)=\left[2^{j+1} I \backslash 2^{j} I\right] \cap(0,1)$ if $j>0$ and $S_{0}(I)=2 I$. Then as in the proof of Theorem 4.4 we decompose $a_{2}$ as follows:

$$
\begin{aligned}
a_{2}= & \sum_{j=k_{0}-3}^{\infty} a_{2} \chi_{S_{j}(I)}+\sum_{j=0}^{k_{0}-3}\left(a_{2} \chi_{S_{j}(I)}-\frac{\chi_{S_{j}(I)}}{\left|S_{j}(I)\right|} \int_{S_{j}(I)} a_{2}\right) \\
& +\sum_{j=0}^{k_{0}-3}\left(\frac{\chi_{S_{j}(I)}}{\left|S_{j}(I)\right|}-\frac{\chi_{S_{j-1}(I)}}{\left|S_{j-1}(I)\right|}\right) \int_{2^{k_{0}-3} \backslash 2^{j} I} a_{2}+\frac{\chi_{2 I}}{|2 I|} \int_{2^{k_{0}-3} I} a_{2} \\
=: & A_{1}+A_{2}+A_{3}+A_{4} .
\end{aligned}
$$

By arguing in a similar way to the proof of Theorem 4.4 we can show that firstly $A_{1}$ can be expressed as an atomic representation of type (b) atoms of Definition 4.24; and secondly that $A_{2}$ and $A_{3}$ can be expressed as an atomic representation of type (a) atoms.

It remains then to take care of $A_{4}$. Firstly note that $\operatorname{supp} A_{4} \subset 2^{k_{0}} I=: \widehat{I}$. Next recall that $q_{s}(x, y)$ is the kernel of $s L e^{-s L}$. Then applying Lemma 4.22 we have

$$
\int_{2^{k_{0}-3} I} a_{2}=\int_{2^{k_{0}-3} I} \int_{0}^{r_{I}^{2}} s L e^{-s L} a(x) \frac{d s}{s} d x \lesssim \int_{0}^{r_{I}^{2}} \frac{s}{\left(2^{k_{0}-3} r_{I}\right)^{2}} \int_{I}|a(y)| d y \frac{d s}{s} \lesssim 2^{-2 k_{0}}|I|^{1-1 / p},
$$

and since $p>1 / 2$ then

$$
\left\|A_{4}\right\|_{L^{\infty}} \lesssim \frac{2^{-2 k_{0}}}{|I|^{1 / p}} \lesssim \frac{1}{\left|2^{k_{0}} I\right|^{1 / p}}=|\widehat{I}|^{-1 / p}
$$

Now since $4 \widehat{I} \cap(0,1)^{c} \neq \emptyset$ we may evoke Case 1 to obtain $\left\|A_{4}\right\|_{H_{a t}^{p}((0,1), d x)} \lesssim 1$.

Step 2: $H_{a t}^{p}((0,1), d x) \subset H_{L, \mathrm{rad}}^{p}((0,1), d x)$. It suffices to prove that there exists $C>0$ so that

$$
\left\|\sup _{t>0}\left|e^{-t L} a\right|\right\|_{L^{p}((0,1), d x)} \leq C
$$

for all $H^{p}((0,1), d x)$-atoms $a$. Now if $a$ is type (b) atom then the inequality (54) follows from part (i) of Lemma 4.23 and so we need only to take care of type (a) atoms.

Therefore we suppose that $a$ is an $H^{p}((0,1), d x)$-atom type (a) supported in an interval $I$. If $I \subset \mathcal{J}_{j}^{*}$ for some $j \in \mathbb{Z}^{*}$, then (54) follows from part (ii) of Lemma 4.23 . Otherwise, if $I \not \subset \mathcal{J}_{j}^{*}$ for any $j \in \mathbb{Z}^{*}$, then there must exist a largest index $j_{1} \in \mathbb{Z}^{*}$ and a smallest index $j_{2} \in \mathbb{Z}^{*}$ so that $j_{1}<j_{2}$ and $I=\sum_{j=j_{1}}^{j_{2}} \mathcal{J}_{j}^{*}$. 
Set $j_{0}:=\min \left\{\left|j_{1}\right|,\left|j_{2}\right|\right\}$ if $j_{1} j_{2}>0$, and $j_{0}:=0$ if $j_{1} j_{2}<0$. Then we have $|I| \sim 2^{-j_{0}}$. We now decompose $a$ as follows:

$$
a=\sum_{j=j_{1}}^{j_{2}} 2^{\left(j_{0}-|j|\right) / p} a_{j}
$$

where

Then supp $a_{j} \subset \mathcal{J}_{j}^{*}$ and

$$
a_{j}=2^{-\left(j_{0}-|j|\right) / p} \frac{\chi_{\mathcal{J}_{j}^{*}}}{\sum_{i \in \mathbb{Z}^{*}} \chi \mathcal{J}_{i}^{*}} a .
$$

$$
\left\|a_{j}\right\|_{L^{\infty}} \lesssim 2^{-\left(j_{0}-|j|\right) / p}\|a\|_{L^{\infty}} \lesssim 2^{-\left(j_{0}-|j|\right) / p}|I|^{-1 / p} \lesssim 2^{-\left(j_{0}-|j|\right) / p_{2} 2^{j_{0} / p}}=2^{|j| / p} \sim\left|\mathcal{J}_{j}^{*}\right|^{-1 / p}
$$

Therefore, if we write

$$
a_{j}=\left[a_{j}-\frac{\chi_{\mathcal{J}_{j}}}{\left|\mathcal{J}_{j}\right|} \int_{\mathcal{J}_{j}^{*}} a_{j}(x) d x\right]+\frac{\chi_{\mathcal{J}_{j}}}{\left|\mathcal{J}_{j}\right|} \int_{\mathcal{J}_{j}^{*}} a_{j}(x) d x=a_{j 1}+a_{j 2}
$$

then from (56) it follows that $a_{j 1}$ is type (a) atom supported in $\mathcal{J}_{j}^{*}$ and that $a_{j 2}$ is an type (b) atom. This along with Lemma 4.23 implies that $\left\|a_{j}\right\|_{H^{p}((0,1), d x)} \lesssim 1$. Then taking into account (55) we see that $\|a\|_{H^{p}((0,1), d x)} \lesssim 1$, completing our proof.

4.5. Fourier-Bessel operators on $\left((0,1), x^{2 \nu+1} d x\right)$. Consider the following differential operator

$$
L=-\frac{d^{2}}{d x^{2}}-\frac{2 \nu+1}{x} \frac{d}{d x}, \quad \nu>-1 .
$$

Let $\left\{\lambda_{k, \nu}\right\}_{k \geq 1}$ denote the sequence of succesive positive zeros of the Bessel function $J_{\nu}$ and consider

$$
\phi_{k}^{\nu}(x)=d_{k, \nu} \lambda_{k, \nu}^{1 / 2} J_{\nu}\left(\lambda_{k, \nu} x\right) x^{-\nu}
$$

where $x \in(0,1)$ and $d_{k, \nu}=\sqrt{2}\left|\lambda_{k, \nu} J_{\nu+1}\left(\lambda_{k, \nu}\right)\right|^{-1}$.

The system $\left\{\phi_{k}^{\nu}\right\}_{k}$ forms an orthornomal basis for $L^{2}((0,1), d \mu)$, where $d \mu(x)=x^{2 \nu+1} d x$. It is well known that

$$
L \phi_{k}^{\nu}(x)=\lambda_{k, \nu}^{2} \phi_{k}^{\nu}(x)
$$

The operator $L$ has a non-negative self-adjoint extensions which is still denoted by $L$ with domain

$$
D(L)=\left\{f \in L^{2}\left((0,1), x^{2 \nu+1} d x\right): \sum_{k=1}^{\infty} \lambda_{k, \nu}^{4}\left|\left\langle f, \phi_{k}^{\nu}\right\rangle\right|^{2}<\infty\right\} .
$$

Let us denote $(X, d, \mu)=\left((0,1),|\cdot|, x^{2 \nu+1} d x\right)$. One can easily show that

$$
\mu(I)= \begin{cases}x^{2 \nu+1} r, & x>r \\ r^{2 \nu+2}, & x \leq r\end{cases}
$$

where $I=(x-r, x+r) \cap(0, \infty)$ with $x \in(0,1)$ and $r<1$. It is clear then that the triple $(X,|\cdot|, d \mu)$ is a space of homogeneous type with dimension $n=2 \nu+2$.

As in [19], we now consider the intervals:

$$
\mathcal{I}_{j}=\left(1-2^{-j}, 1-2^{-j-1}\right], \quad j=0,1, \ldots
$$

which are depicted in Figure 2. It is obvious that the family $\left\{\mathcal{I}_{j}\right\}_{j \in \mathbb{N}}$ is pairwise disjoint and $(0,1)=\bigcup_{j \in \mathbb{N}} \mathcal{I}_{j}$. For each $j \in \mathbb{N}$ we shall denote by $\mathcal{I}_{j}^{*}=\frac{1}{20} \mathcal{I}_{j}$ and $\mathcal{I}_{j}^{* *}=\frac{1}{10} \mathcal{I}_{j}$.

Consider the following atoms.

Definition 4.24. Let $p \in\left(\frac{2 \nu+2}{2 \nu+3}, 1\right]$. A function a is a type (a) atom associated with an interval $I \subseteq(0,1)$ if

(i) $\operatorname{supp} a \subset I$

(ii) $\|a\|_{L^{\infty}} \leq \mu(I)^{-1 / p}$

(iii) $\int a(x) d \mu(x)=0$ 
A function $a$ is a type (b) atom if

for some $j \in \mathbb{N}$.

$$
a(x)=\frac{\chi_{\mathcal{I}_{j}}(x)}{\mu\left(\mathcal{I}_{j}\right)^{1 / p}}
$$

We say a function a is an $H^{p}((0,1), d \mu)$-atom associated with $I$ if it is either a type (a) or type (b) atom.

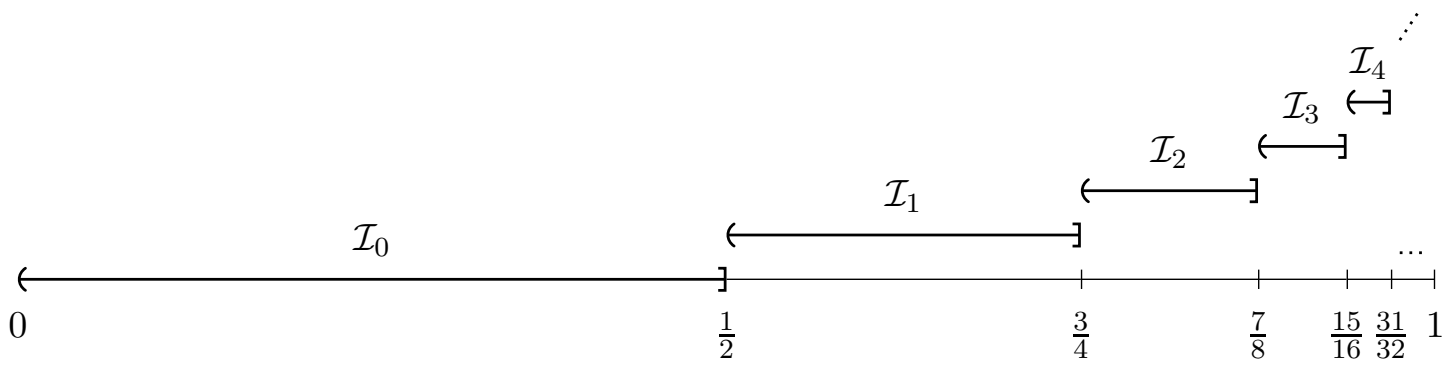

FiguRE 2. Intervals for $\mathcal{I}_{j}$

Definition 4.25 (Atomic Hardy spaces on $((0,1), d \mu))$. Given $p \in\left(\frac{2 \nu+2}{2 \nu+3}, 1\right]$, we say that $f=$ $\sum \lambda_{j} a_{j}$ is an atomic $H^{p}((0,1), d \mu)$-representation if $\left\{\lambda_{j}\right\}_{j=0}^{\infty} \in l^{p}$, each $a_{j}$ is a $H^{p}((0,1), d \mu)$ atom, and the sum converges in $L^{2}(X)$. The space $H_{a t}^{p}((0,1), d \mu)$ is then defined as the completion of

with the norm given by

$$
\left\{f \in L^{2}: f \text { has an atomic } H^{p}((0,1), d \mu) \text {-representation }\right\},
$$

$\|f\|_{H_{a t}^{p}((0,1), d \mu)}=\inf \left\{\left(\sum\left|\lambda_{j}\right|^{p}\right)^{1 / p}: f=\sum \lambda_{j} a_{j}\right.$ is an atomic $H^{p}((0,1), d \mu)$-representation $\}$.

As in Definition $(1.3)$ we denote by $H_{L, \text { rad }}^{p}((0,1), d \mu)$ and $H_{L, \max }^{p}((0,1), d \mu)$ respectively the maximal Hardy spaces defined via radial and non-tangential maximal functions associated to $L$.

Our main result in this section is the following:

Theorem 4.26. Let $\nu>-1$.

For $p \in\left(\frac{2 \nu+2}{2 \nu+3}, 1\right]$ we have

$$
H_{L, \operatorname{rad}}^{p}((0,1), d \mu) \equiv H_{L, \max }^{p}((0,1), d \mu) \hookrightarrow H_{a t}^{p}((0,1), d \mu) .
$$

In the particular case $p=1$, we have

$$
H_{L, \mathrm{rad}}^{1}((0,1), d \mu) \equiv H_{L, \max }^{1}((0,1), d \mu) \equiv H_{a t}^{1}((0,1), d \mu)
$$

with equivalent norms.

Remark 4.27. It was proved in [19] that

$$
H_{a t}^{1}((0,1), d \mu) \equiv H_{\sqrt{L}, \text { rad }}^{1}((0,1), d \mu) \equiv H_{\sqrt{L}, \max }^{1}((0,1), d \mu)
$$

under the restriction $\nu>-1 / 2$. In this situation, Theorem 4.26 gives a maximal function characterization for the Hardy space $H_{a t}^{1}((0,1), d \mu)$ for the full range of $\nu$.

Before we give the proof of Theorem 4.26 we need to develop some technical material. We shall continue to use the notion of "interval" as expressed in (51).

We define the critical function for $L$ by

$$
\rho(x):=\frac{1}{3}(1-x), \quad x \in(1 / 2,1)
$$

By a critical interval $I_{\rho}$ we mean an interval with centre $x_{I} \in(1 / 2,1)$ and radius $\rho_{I}:=\rho\left(x_{I}\right)$.

Our critical intervals admit the following desirable properties, whose easy proofs we omit. 
Lemma 4.28. Let $I_{\rho}$ be a critical interval related to the function $\rho$ from (59).

(a) If $y \in I_{\rho}$ then $\frac{2}{3} \rho_{I}<\rho(y)<\frac{4}{3} \rho_{I}$.

(b) If $x, y \in I_{\rho}$ then $\frac{1}{2} \rho(x)<\rho(y)<2 \rho(x)$.

We emphasize that the restriction $x_{I} \in(1 / 2,1)$ for a critical interval is essential in Lemma 4.28. It is not difficult to see that these estimates are invalid if we allow $x_{I}$ to be near the endpoint 0 .

We next have a useful integration by parts lemma.

Lemma 4.29. Let $f, g$ be sufficiently smooth functions on $X$ and suppose either $f^{\prime}(1)=0$ or $g(1)=0$ holds. Then we have

$$
\int_{0}^{1} L f(x) g(x) d \mu(x)=\int_{0}^{1} f^{\prime}(x) g^{\prime}(x) d \mu(x) .
$$

Proof. From integration by parts and the conditions on $f$ and $g$,

$$
\begin{aligned}
\int_{0}^{1} L f(x) g(x) d \mu(x)= & -\left.f^{\prime}(x) g(x) x^{2 \nu+1}\right|_{0} ^{1}+\int_{0}^{1} f^{\prime}(x)\left(g(x) x^{2 \nu+1}\right)^{\prime} d x \\
& -\int_{0}^{1} \frac{2 \nu+1}{x} f^{\prime}(x) g(x) x^{2 \nu+1} d x \\
= & \int_{0}^{1} f^{\prime}(x) g^{\prime}(x) x^{2 \nu+1} d x+\int_{0}^{1} f^{\prime}(x) g(x)\left(x^{2 \nu+1}\right)^{\prime} d x \\
& -\int_{0}^{1} \frac{2 \nu+1}{x} f^{\prime}(x) g(x) x^{2 \nu+1} d x \\
= & \int_{0}^{1} f^{\prime}(x) g^{\prime}(x) x^{2 \nu+1} d x
\end{aligned}
$$

We now collect together some estimates on the kernels related to $L$. Let $p_{t}(x, y)$ be the kernel of the heat semigroup $e^{-t L}$ associated with $L$. Then one has the following two sided bounds.

Lemma 4.30 ([28] Theorem 1.1). For $\nu>-1$ we have

$$
p_{t}(x, y) \approx \frac{(1+t)^{\nu+2}}{(t+x y)^{\nu+1 / 2}}\left(1 \wedge \frac{(1-x)(1-y)}{t}\right) \frac{1}{\sqrt{t}} e^{-\frac{|x-y|^{2}}{4 t}-\lambda_{1, \nu}^{2} t}
$$

for $x, y \in X$ and $t>0$.

Lemma 4.30 and a simple calculation yields

$$
p_{t}(x, y) \lesssim \frac{1}{\mu\left(I_{\sqrt{t}}(x)\right)}\left(1 \wedge \frac{(1-x)(1-y)}{t}\right) e^{-\frac{|x-y|^{2}}{c t}}
$$

for all $x, y \in X$ and $t>0$.

Denote by $q_{t}(x, y)$ the kernel of $t L e^{-t L}$. It is well-known that the heat kernel bounds (60) can be transfered to the kernel $q_{t}(x, y)$. That is, we have

$$
q_{t}(x, y) \lesssim \frac{1}{\mu\left(I_{\sqrt{t}}(x)\right)} e^{-\frac{|x-y|^{2}}{c t}}
$$

for all $x, y \in X$ and $t>0$.

Lemma 4.31. For $\nu>-1$ we have

$$
\left|\partial_{x} p_{t}(x, y)\right| \lesssim \frac{1}{(x y)^{\nu+1 / 2}} \frac{e^{-(x-y)^{2} / 4 t}}{t}
$$

for all $x, y \in X$ and $t>0$. 
Proof. Let $\tilde{p}_{t}(x, y)$ be the heat kernel of the operator Fourier-Bessel operator $(46)$ on $((0,1), d x)$. It is well known that the heat kernels $\tilde{p}_{t}(x, y)$ and $p_{t}(x, y)$ are related through (see [19])

$$
\tilde{p}_{t}(x, y)=(x y)^{\nu+1 / 2} p_{t}(x, y) \text {. }
$$

It follows that

$$
(x y)^{\nu+1 / 2} \partial_{x} p_{t}(x, y)=\left(\partial_{x}-\frac{\nu+1 / 2}{x}\right) \tilde{p}_{t}(x, y)
$$

and one can now follow the argument in [19, Lemma 2.4] to obtain

$$
\left|\left(\partial_{x}-\frac{\nu+1 / 2}{x}\right) \tilde{p}_{t}(x, y)\right| \lesssim \frac{e^{-(x-y)^{2} / 4 t}}{t}
$$

This yields (62) as desired.

We now invest Lemma 4.31 to obtain the following analogue of Lemma 4.22.

Lemma 4.32. (a) Let I with $x_{I} \in(0,1 / 2)$ and $r_{I} \geq 1 / 8$. Then we have

$$
\left|\int_{I} q_{t}(x, y) d \mu(x)\right| \lesssim \frac{t^{1 / 4} e^{-c r_{I}^{2} / t} \mu(I)^{1 / 2}}{y^{\nu+1 / 2}} \quad \forall y \in \frac{1}{2} I
$$

for any $t>0$.

(b) For any critical interval $I_{\rho}$ we have

$$
\left|\int_{I_{\rho}} q_{t}(x, y) d \mu(x)\right| \lesssim \frac{t^{1 / 4}}{\sqrt{\rho_{I}}} e^{-c \rho_{I}^{2} / t}, \quad \forall y \in \frac{1}{2} I_{\rho}
$$

for any $t>0$.

Proof. We first prove (a). Choose a cutoff function $\varphi \in C_{c}^{\infty}(X)$ supported in $I$ with $0 \leq \varphi \leq 1$, equal to 1 on $\frac{3}{4} I$ and with $\left|\varphi^{\prime}(x)\right| \lesssim 1 / r_{I}$. Then

$$
\left|\int_{I} q_{t}(x, y) d \mu(x)\right| \lesssim\left|\int_{X} t \partial_{t} p_{t}(x, y) \varphi(x) d \mu(x)\right|+\left|\int_{I \backslash \frac{3}{4} I} q_{t}(x, y)[1-\varphi(x)] d \mu(x)\right|=: I_{1}+I_{2} .
$$

For the term $I_{2}$, using (61) and that $|x-y| \sim r_{I}$, we have

$$
I_{2} \lesssim \int_{I \backslash \frac{3}{4} I} \frac{1}{\mu\left(I_{\sqrt{t}}(x)\right)} e^{-\frac{|x-y|^{2}}{c t}} d \mu(x) \lesssim e^{-\frac{r_{I}^{2}}{c^{\prime} t}} \int_{I \backslash \frac{3}{4} I} \frac{1}{\mu\left(I_{\sqrt{t}}(x)\right)} e^{-\frac{|x-y|^{2}}{2 c t}} d \mu(x) \lesssim e^{-\frac{r_{I}^{2}}{c^{\prime} t}}
$$

For the term $I_{1}$ since $\partial_{t} p_{t}(\cdot, y)=-L p_{t}(\cdot, y)$, then by Lemma 4.29 , estimate $(62)$ and the fact that $\left|\varphi^{\prime}(x)\right| \lesssim r_{I}^{-1}$, we obtain

$$
I_{1}=\left|\int_{X} t \partial_{x} p_{t}(x, y) \partial_{x} \varphi(x) d \mu(x)\right| \lesssim \frac{1}{r_{I}}\left|\int_{\frac{3}{4} I} \frac{1}{(x y)^{\nu+1 / 2}} e^{-(x-y)^{2} / c t} d \mu(x)\right| .
$$

Hölder's inequality and the estimate $|x-y| \sim r_{I}$ then gives

$$
\begin{aligned}
I_{1} & \lesssim \frac{1}{r_{I}} \mu(I)^{1 / 2}\left[\int_{\frac{3}{4} I} \frac{1}{(x y)^{2 \nu+1}} e^{-(x-y)^{2} / c^{\prime} t} d \mu(x)\right]^{1 / 2} \\
& \lesssim \frac{t^{1 / 4}}{r_{I}} \mu(I)^{1 / 2} e^{-c r_{I}^{2} / t}\left[\int_{\frac{3}{4} I} \frac{1}{y^{2 \nu+1}} \frac{1}{\sqrt{t}} e^{-(x-y)^{2} / 2 c^{\prime} t} d x\right]^{1 / 2} \\
& \lesssim \frac{t^{1 / 4}}{r_{I}} \frac{\mu(I)^{1 / 2} e^{-c r_{I}^{2} / t}}{y^{\nu+1 / 2}} .
\end{aligned}
$$

The condition $r_{I} \geq 1 / 8$ allows us to conclude

$$
I_{1} \lesssim \frac{t^{1 / 4} e^{-c r_{I}^{2} / t} \mu(I)^{1 / 2}}{y^{\nu+1 / 2}} .
$$

This and the estimate of $I_{2}$ proves (a). 
If $I_{\rho}$ is a critical interval, then we have $y \sim x_{I}$ and $\mu(I) \sim x_{I}^{2 \nu+1} \rho_{I}$. Hence, (63) implies

$$
I_{1} \lesssim \frac{t^{1 / 4}}{\sqrt{\rho_{I}}} e^{-c \rho_{I}^{2} / t}
$$

Part (b) now follows by combining this estimate with the estimate of $I_{2}$ from (a).

We can now give the analogue of Lemma 4.23 for $p=1$. Note that the intervals $\mathcal{I}_{j}^{*}$ and $\mathcal{I}_{j}^{* *}$ have been defined in the comments after (58).

Lemma 4.33. Let that $\nu>-1$. Suppose that a is either

(i) a type-(b) $H^{1}((0,1), d \mu)$-atom, or

(ii) a type-(a) $H^{1}((0,1), d \mu)$-atom supported in $\mathcal{I}_{j}^{*}$ for some $j \in \mathbb{N}$.

Then there exists $C>0$ independent of a so that

$$
\left\|\sup _{t>0}\left|e^{-t L} a\right|\right\|_{L^{1}((0,1), d \mu)} \leq C .
$$

Proof of Lemma 4.33. Part (i). Suppose that $a$ is an $H^{1}((0,1), d \mu)$-atom of type-(b). Then $a=\mu\left(\mathcal{I}_{j}\right)^{-1} \chi_{\mathcal{I}_{j}}$ for $j \geq 0$. Since the radial maximal operator associated with $L$ is uniformly bounded on $L^{\infty}$ then we have, for $j=0,1,2$,

$$
\left\|\sup _{t>0}\left|e^{-t L} a\right|\right\|_{L^{1}((0,1), d \mu)} \leq\|a\|_{L^{\infty}}=\mu\left(\mathcal{I}_{j}\right) \lesssim 1 .
$$

In a similar way we have, for $j \geq 3$,

$$
\left\|\sup _{t>0}\left|e^{-t L} a\right|\right\|_{L^{1}\left(3 \mathcal{I}_{j}, d \mu\right)} \lesssim 1
$$

and so it remains to show

$$
\left\|\sup _{t>0}\left|e^{-t L} a\right|\right\|_{L^{1}\left(\left(3 \mathcal{I}_{j}\right)^{c}, d \mu\right)} \lesssim 1
$$

Firstly note that if $y \in \mathcal{I}_{j}$ then we have the estimates $y \sim 1$ and $1-y \sim r_{\mathcal{I}_{j}} \sim \mu\left(\mathcal{I}_{j}\right) \sim 2^{-j}$. Secondly if $x \in\left(3 \mathcal{I}_{j}\right)^{c}$ then we also have $|x-y| \sim 1-x$. These facts along with Lemma 4.30 gives

$$
\begin{aligned}
\left\|\sup _{t>0}\left|e^{-t L} a\right|\right\|_{L^{1}\left(\left(3 \mathcal{I}_{j}\right)^{c}, d \mu\right)} & \lesssim \mu\left(\mathcal{I}_{j}\right)^{-1} \int_{\left(3 \mathcal{I}_{j}\right)^{c}} \sup _{t>0} \int_{\mathcal{I}_{j}} \frac{(1-x)}{(t+x)^{\nu+1 / 2}} \frac{2^{-j}}{t^{3 / 2}} e^{-\frac{(1-x)^{2}}{c t}} d y d \mu(x) \\
& \lesssim \int_{\left(3 \mathcal{I}_{j}\right)^{c}} \frac{2^{-j} x^{\nu+1 / 2}}{(1-x)^{2}} d x
\end{aligned}
$$

Now since $\left(3 \mathcal{I}_{j}\right)^{c}=\left(0,1-3 \times 2^{-j}\right)$ then a direct calculation gives (64).

Part (ii). Suppose that $a$ is an $H^{1}((0,1), d \mu)$-atom of type-(a) associated to an interval $I \subset \mathcal{I}_{j}^{*}$ for some $j \in \mathbb{N}$.

We now consider two cases: $j \geq 1$ and $j=0$.

Case 1: $j \geq 1$. In a similar fashion to part (ii) of Lemma 4.23, it suffices to prove that

$$
\left\|\sup _{t>0}\left|e^{-t L} a\right|\right\|_{L^{1}\left((2 I)^{c}, d \mu\right)} \lesssim 1
$$

In this situation we have $x \sim y \sim 1$ for every $x \in 3 \mathcal{I}_{j}$ and $y \in I$. This fact, the cancellation property of $a$, and Lemma 4.31 imply that

$$
\begin{aligned}
\left\|\sup _{t>0}\left|e^{-t L} a\right|\right\|_{L^{1}\left(\left(3 \mathcal{I}_{j} \backslash 2 I\right), d \mu\right)} & \\
& =\int_{3 \mathcal{I}_{j} \backslash 2 I} \sup _{t>0}\left|\int_{I}\left[p_{t}(x, y)-p_{t}\left(x, x_{I}\right)\right] a(y) d \mu(y)\right| d \mu(x) \\
& \lesssim \int_{3 \mathcal{I}_{j} \backslash 2 I} \sup _{t>0} \int_{I} \frac{\left|y-x_{I}\right|}{(x y)^{\nu+1 / 2}} \frac{e^{-(x-y)^{2} / c t}}{t}|a(y)| d \mu(y) d \mu(x) .
\end{aligned}
$$


Since $d \mu(x) \sim d x$ whenever $x \in 3 \mathcal{I}_{j}$, and $|x-y| \sim\left|x-x_{I}\right|$ whenever $x \in(2 I)^{c}$ and $y \in I$, we may continue with

$$
\left\|\sup _{t>0}\left|e^{-t L} a\right|\right\|_{L^{1}\left(\left(3 \mathcal{I}_{j} \backslash 2 I\right), d \mu\right)} \lesssim \int_{3 \mathcal{I}_{j} \backslash 2 I} \sup _{t>0} \int_{I} \frac{r_{I}}{t} e^{-\left|x-x_{I}\right|^{2} / c t}|a(y)| d \mu(y) d x \lesssim \int_{3 \mathcal{I}_{j} \backslash 2 I} \frac{r_{I}}{\left|x-x_{I}\right|^{2}} d x
$$

which evaluates to

$$
\left\|\sup _{t>0}\left|e^{-t L} a\right|\right\|_{L^{1}\left(3 \mathcal{I}_{j} \backslash 2 I\right)} \lesssim 1 .
$$

Since $y \sim 1$ for $y \in I \subset 3 \mathcal{I}_{j}$ then arguing similarly to (66) we have

$$
\begin{aligned}
\left\|\sup _{t>0}\left|e^{-t L} a\right|\right\|_{L^{1}\left(\left(3 \mathcal{I}_{j}\right)^{c}, d \mu\right)} & \lesssim \int_{\left(3 \mathcal{I}_{j}\right)^{c}} \sup _{t>0} \int_{I} \frac{\left|y-x_{I}\right|}{(x y)^{\nu+1 / 2}} \frac{1}{t} e^{-(x-y)^{2} / c t}|a(y)| d \mu(y) d \mu(x) \\
& \lesssim \int_{\left(3 \mathcal{I}_{j}\right)^{c}} \sup _{t>0} \int_{I} \frac{r_{I}}{x^{\nu+1 / 2}} \frac{1}{t} e^{-\left|x-x_{I}\right|^{2} / c t}|a(y)| d \mu(y) d \mu(x) \\
& \lesssim \int_{\left(3 \mathcal{I}_{j}\right)^{c}} \frac{r_{I}}{\left|x-x_{I}\right|^{2}} x^{\nu+1 / 2} d x .
\end{aligned}
$$

Noting that $\left(3 \mathcal{I}_{j}\right)^{c}=\left(0,1-3 \times 2^{-j}\right)$, and applying the estimates $r_{I} \lesssim 2^{-j}$ and $\left|x-x_{I}\right| \sim|1-x|$ whenever $x \in\left(3 \mathcal{I}_{j}\right)^{c}$ we continue with

$$
\left\|\sup _{t>0}\left|e^{-t L} a\right|\right\|_{L^{1}\left(\left(3 \mathcal{I}_{j}\right)^{c}, d \mu\right)} \lesssim \int_{0}^{1-3 \times 2^{-j}} \frac{2^{-j} x^{\nu+1 / 2}}{|1-x|^{2}} d x \lesssim 1 .
$$

Estimate (68) and (67) together gives (65).

Case 2: $j=0$. We write

$$
\begin{aligned}
& \left\|\sup _{t>0}\left|e^{-t L} a\right|\right\|_{L^{1}((0,1), d \mu)} \\
& \quad \leq\left\|\sup _{t \geq 1}\left|e^{-t L} a\right|\right\|_{L^{1}((0,1), d \mu)}+\left\|\sup _{0<t<1}\left|e^{-t L} a\right|\right\|_{L^{1}\left(\mathcal{I}_{0}^{* *}\right)}+\left\|\sup _{0<t<1}\left|e^{-t L} a\right|\right\|_{L^{1}\left(\left(\mathcal{I}_{0}^{* *}\right)^{c}\right)} \\
& \quad=: A_{1}+A_{2}+A_{3} .
\end{aligned}
$$

From Lemma 4.30 we see that the heat kernel is dominated by 1 whenever $t \geq 1$, so as a consequence we obtain easily that $A_{1} \lesssim\|a\|_{L^{1}} \lesssim 1$. Again from Lemma 4.30 and the fact that $|x-y| \sim x \sim 1$ we have

$$
A_{3} \lesssim \int_{\left(\mathcal{I}_{0}^{* *}\right)^{c}} \sup _{0<t<1} \int_{I} \frac{1}{(t+y)^{\nu+1 / 2}} \frac{1}{\sqrt{t}} e^{-\frac{c}{t}}|a(y)| d \mu(y) d \mu(x) .
$$

Since the inner integrand can be controlled by a constant multiple of $|a(y)|$ for all $t \in(0,1)$ and $y \in I$, then we obtain $A_{3} \lesssim\|a\|_{L^{1}} \lesssim 1$.

To handle $A_{2}$ we shall employ a comparison with a Bessel operator on $((0, \infty), d \mu)$ defined by

$$
\mathfrak{L}=-\frac{d^{2}}{d x^{2}}-\frac{2 \nu+1}{x} \frac{d}{d x} .
$$

Let $h_{t}(x, y)$ be the kernel of $e^{-t \mathfrak{L}}$. Then it is well-known that

$$
\begin{aligned}
h_{t}(x, y) & \lesssim \frac{1}{\mu\left(I_{\sqrt{t}}(x)\right)} e^{-\frac{|x-y|^{2}}{c t}} \\
\left|\partial_{x} h_{t}(x, y)\right| & \lesssim \frac{1}{\sqrt{t} \mu\left(I_{\sqrt{t}}(x)\right)} e^{-\frac{|x-y|^{2}}{c t}}
\end{aligned}
$$

We now split the term $A_{2}$ as follows:

$$
A_{2} \leq\left\|\sup _{0<t<1}\left|\left(e^{-t L}-e^{-t \mathfrak{L}}\right) a\right|\right\|_{L^{1}\left(\mathcal{I}_{0}^{* *}\right)}+\left\|\sup _{0<t<1} \mid e^{-t \mathfrak{L} a \mid}\right\|_{L^{1}\left(\mathcal{I}_{0}^{* *}\right)}=: A_{21}+A_{22} .
$$


From (69) and (70) above, by a standard argument we have

$$
A_{22} \leq\left\|\sup _{t>0}\left|e^{-t \mathfrak{L}} a\right|\right\|_{L^{1}(0, \infty)} \lesssim 1 .
$$

On the other hand, Corollary 4.7 in [19] shows that $A_{21} \lesssim\|a\|_{L^{1}} \lesssim 1$ and hence we have $A_{2} \lesssim 1$.

Taking the estimates of $A_{1}, A_{2}, A_{3}$ into account, we arrive at the required estimate as stated in the Lemma. This completes our proof.

We are now ready to prove Theorem 4.26, which is a direct consequence of Theorem 1.4 and the following proposition.

Proposition 4.34. Let $\nu>-1$. Then for $p \in\left(\frac{2 \nu+2}{2 \nu+3}, 1\right]$ we have

(i) $H_{L, \text { rad }}^{p}(X) \subset H_{a t}^{p}(X)$;

(ii) $H_{a t}^{1}(X) \subset H_{L, \mathrm{rad}}^{1}(X)$.

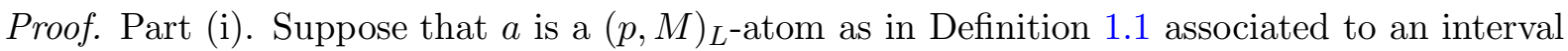
$I$. We consider two cases: $4 I \cap[1, \infty) \neq \emptyset$ and $4 I \subset(0,1)$.

Case 1: $4 I \cap[1, \infty) \neq \emptyset$. In this situation, it easy to see that if $x_{I} \in \mathcal{I}_{j}$ for some $j \geq 0$, then $I \subset \mathcal{I}_{j-1} \cup \mathcal{I}_{j} \cup \mathcal{I}_{j+1}$ and $\mu(I) \sim \mu\left(\mathcal{I}_{j}\right)$. Hence, using the decomposition

$$
a=\left[a-\frac{\chi_{\mathcal{I}_{j}}}{\mu\left(\mathcal{I}_{j}\right)} \int a\right]+\frac{\chi_{\mathcal{I}_{j}}}{\mu\left(\mathcal{I}_{j}\right)} \int a=: \tilde{a}_{1}+\tilde{a}_{2}
$$

we see that $a_{1}$ is a type-(a) atom, while $a_{2}$ is a type-(b) atom from Definition 4.24. Hence, $a \in H_{a t}^{p}((0,1), d \mu)$.

Case 2: $4 I \subset(0,1)$. In this case, $a$ can be expressed in the form $a=L b$. We now write

$$
a=L e^{-r_{I}^{2} L} b+L\left(I-e^{-r_{I}^{2} L}\right) b=L e^{-r_{I}^{2} L} b+\left(I-e^{-r_{I}^{2} L}\right) a=a_{1}+a_{2}
$$

where $b$ is supported in $B$ and satisfies

$$
\|b\|_{L^{\infty}} \leq r_{I}^{2} \mu(I)^{-1 / p} .
$$

At this stage the proof is similar to that of Theorem 4.4 and we will just sketch the main ideas.

We take care of $a_{2}$ only, since $a_{1}$ can be treated similarly. We choose $k_{0} \in \mathbb{N}$ so that $2^{k_{0}-1} r_{I} \leq$ $1-x_{I}<2^{k_{0}} r_{I}$. Hence $k_{0} \geq 3$. We set $S_{j}(I)=\left[2^{j+1} I \backslash 2^{j} I\right] \cap(0,1)$ if $j>0$ and $S_{0}(I)=2 I$. Then as in the proof of Theorem 4.4 we decompose $a_{2}$ as follows:

$$
\begin{aligned}
a_{2}= & \sum_{j=k_{0}-3}^{\infty} a_{2} \chi_{S_{j}(I)}+\sum_{j=0}^{k_{0}-3}\left(a_{2} \chi_{S_{j}(I)}-\frac{\chi_{S_{j}(I)}}{\mu\left(S_{j}(I)\right)} \int_{S_{j}(I)} a_{2}\right) \\
& +\sum_{j=0}^{k_{0}-3}\left(\frac{\chi_{S_{j}(I)}}{\mu\left(S_{j}(I)\right)}-\frac{\chi_{S_{j-1}(I)}}{\mu\left(S_{j-1}(I)\right)}\right) \int_{2^{k_{0}-3} \backslash 2^{j} I} a_{2}+\frac{\chi_{2 I}}{\mu(2 I)} \int_{2^{k_{0}-3} I} a_{2} \\
=: & A_{1}+A_{2}+A_{3}+A_{4} .
\end{aligned}
$$

Arguing similarly to the proof of Theorem 4.4 we can show that $A_{1}, A_{2}$ and $A_{3}$ can each be expressed as atomic representations of atoms from Definition $4.24 ; A_{1}$ as type-(b) atoms, $A_{2}$ and $A_{3}$ as type-(a) atoms.

It remains to take care of $A_{4}$. We consider two subcases: $x_{I} \leq 1 / 2$ and $x_{I}>1 / 2$.

Subcase 2.1: $x_{I} \in(0,1 / 2]$. In this situation we have $2^{k_{0}} I=(0,1)$ and hence $2^{k_{0}-3} r_{I} \geq 1 / 8$. We now have

$$
\begin{aligned}
\int_{2^{k_{0}-3} I} a_{2} & =\int_{0}^{r_{I}^{2}} \int_{I} \int_{2^{k_{0}-3} I} q_{s}(x, y) a(y) d \mu(x) d \mu(y) \frac{d s}{s} \\
& \lesssim \mu\left(2^{k_{0}-3} I\right)^{1 / 2} \int_{0}^{r_{I}^{2}} s^{1 / 4} e^{-c 2^{2 k_{0}} r_{I}^{2} / s} \int_{I} \frac{a(y)}{y^{\nu+1 / 2}} d \mu(y) \frac{d s}{s} \\
& \lesssim e^{-c 2^{2 k_{0}}} \mu\left(2^{k_{0}-3} I\right)^{1 / 2} \int_{I} \frac{a(y)}{y^{\nu+1 / 2}} d \mu(y)
\end{aligned}
$$


where in the second inequality we used (a) in Lemma 4.32. By Hölder's inequality we have

$$
\int_{I} \frac{a(y)}{y^{\nu+1 / 2}} d \mu(y) \leq \mu(I)^{1 / 2}\left[\int_{I} \frac{|a(y)|^{2}}{y^{2 \nu+1}} d \mu(y)\right]^{1 / 2} \lesssim \mu(I)^{1 / 2-1 / p}
$$

and so we may continue with

$$
\int_{2^{k_{0}-3} I} a_{2} \lesssim e^{-c 2^{2 k_{0}}} \mu\left(2^{k_{0}-3} I\right)^{1 / 2} \mu(I)^{1 / 2-1 / p} .
$$

Now since $2^{k_{0}} I=(0,1)$, then $\mu\left(2^{k_{0}-3} I\right) \sim 1 \sim \mu\left(\mathcal{I}_{0}\right)$, and so we have

$$
\left\|A_{4}\right\|_{L^{\infty}} \lesssim \frac{e^{-c 2^{2 k_{0}}} \mu\left(2^{k_{0}-3} I\right)^{1 / 2} \mu(I)^{1 / 2-1 / p}}{\mu(I)} \lesssim \frac{1}{\mu\left(2^{k_{0}-3} I\right)^{1 / p}} \sim \frac{1}{\mu\left(\mathcal{I}_{0}\right)^{1 / p}} .
$$

We now decompose $A_{4}$ as follows:

$$
A_{4}=\left[A_{4}-\frac{\chi_{\mathcal{I}_{0}}}{\mu\left(\mathcal{I}_{0}\right)} \int A_{4}\right]+\frac{\chi_{\mathcal{I}_{0}}}{\mu\left(\mathcal{I}_{0}\right)} \int A_{4}=: A_{41}+A_{42} .
$$

Then $A_{41}$ is an $H^{p}((0,1), d \mu)$ of type-(a) and $A_{42}$ is an $H_{a t}^{p}((0,1), d \mu)$-atom of type-(b). We conclude therefore that $\left\|A_{4}\right\|_{H_{a t}^{p}((0,1), d \mu)} \lesssim 1$.

Subcase 2.2: $x \in(1 / 2,1)$. We may repeat the argument in Subcase 2.1 but make use of Lemma 4.32 (b) rather than Lemma 4.32 (a). This completes the proof of part (i).

Part (ii). The argument from Step 2 of Theorem 4.17 carries over harmlessly but with Lemma 4.33 in place of Lemma 4.23. We leave the details to the interested reader.

Acknowledgement. T. A. Bui and X. T Duong were supported by the research grant ARC DP140100649 from the Australian Research Council.

\section{REFERENCES}

[1] P. Auscher, X.T. Duong and A. McIntosh, Boundedness of Banach space valued singular integral operators and Hardy spaces, unpublished manuscript.

[2] P. Auscher, A. McIntosh and E. Russ, Hardy spaces of differential forms on Riemannian manifolds. J. Geom. Anal. 18 (2008), no. 1, 192-248.

[3] P. Auscher and E. Russ, Emmanuel Hardy spaces and divergence operators on strongly Lipschitz domains of $\mathbb{R}^{n}$, J. Funct. Anal. 201 (2003), no. 1, 148-184.

[4] T.A. Bui, X.T. Duong and F.K. Ly, Maximal function characterizations for new local Hardy type spaces on spaces of homogeneous type, Trans. Amer. Math. Soc., to appear.

[5] D. Burkholder, R.F. Gundy and M.L. Silverstein, A maximal function characterization of the class $H^{p}$, Trans. Amer. Math. Soc. 157 (1971), 137-153.

[6] F. Cacciafesta and P. D'Ancona, Weighted $L^{p}$ estimates for powers of self-adjoint operators, Adv. Math. 229 (2012), 501-530.

[7] A. Calderón, An atomic decomposition of distributions in parabolic $H^{p}$ spaces, Adv. Math. 25 (1977), no. $3,216-225$.

[8] A. Calderón and A. Torchinsky, Parabolic maximal functions associated with a distribution, Adv. Math. 16 (1975), 1-64.

[9] D-C. Chang, S.G. Krantz, and E.M. Stein, $H^{p}$ theory on a smooth domain in $\mathbb{R}^{n}$ and elliptic boundary value problems, J. Funct. Anal. 114 (1993), 286-347.

[10] D-C. Chang, Z. Fu, D. Yang, and S. Yang, Real-variable characterizations of Musielak-Orlicz-Hardy spaces associated with Schrödinger operators on domains, Math. Methods Appl. Sci. 39 (2016), no. 3, 533-569.

[11] R. Coifman, A real variable characterization of $H^{p}$, Studia Math. 51 (1974), 269-274.

[12] R.R. Coifman and G. Weiss, Extensions of Hardy spaces and their use in analysis, Bull. Amer. Math. Soc. 83 (1977), 569-645.

[13] T. Coulhon and A. Sikora, Gaussian heat kernel upper bounds via Phragmén-Lindelöf theorem, Proc. Lond. Math. Soc. 96 (2008), 507-544.

[14] S. Dekel, G. Kerkyacharian, G. Kyriazis and P. Petrushev, Hardy spaces associated with non-negative self-adjoint operators, Studia Math. 239 (2017), no. 1, 17-54.

[15] X.T. Duong, S. Hofmann, D. Mitrea, M. Mitrea and L. Yan, Hardy spaces and regularity for the inhomogeneous Dirichlet and Neumann problems, Rev. Mat. Iberoam. 29 (2013), no. 1, 183-236. 
[16] X.T. Duong and A. McIntosh, The $L^{p}$ boundedness of Riesz transforms associated with divergence form operators, Joint Australian-Taiwanese Workshop on Analysis and Applications, Proceedings of the Centre for Mathematics and its Applications 37 (1999), 15-25.

[17] X.T. Duong and L. Yan, Duality of Hardy and BMO spaces associated with operators with heat kernel bounds, J. Amer. Math. Soc. 18 (2005), 943-973.

[18] J. Dziubański, G. Garrigós, T. Martínez, J.L. Torrea and J. Zienkiewicz, BMO spaces related to Schrödinger operators with potentials satisfying a reverse Hölder inequality, Math. Z. 249 (2005), no. 2, 329-356.

[19] J. Dziubanski, M. Preisner, L. Roncal and P. Stinga, Hardy spaces for Fourier-Bessel expansions, J. D'Analyse Mathématique. 128 (2016), 261-287.

[20] J. Dziubański and J. Zienkiewicz, $H^{p}$ spaces associated with Schrödinger operators with potentials from reverse Hölder classes, Colloq. Math. 98 (2003), no. 1, 5-38.

[21] C. Fefferman and E.M. Stein, $H^{p}$ spaces of several variables, Acta Math. 129 (1972), 137-193.

[22] S. Hofmann, G. Lu, D. Mitrea, M. Mitrea and L. Yan, Hardy spaces associated to non-negative self-adjoint operators satisfying Davies-Gaffney estimates, Mem. Amer. Math. Soc. 214 (2011).

[23] S. Hofmann and S. Mayboroda, Hardy and BMO spaces associated to divergence form elliptic operators, Math. Ann. 344 (2009), no. 1, 37-116.

[24] R. Jiang and D. Yang, Orlicz-Hardy spaces associated with operators satisfying Davies-Gaffney estimates, Commun. Contemp. Math. 13 (2011), 331-373.

[25] R.H. Latter, A characterization of $H^{p}\left(\mathbb{R}^{n}\right)$ in terms of atoms, Studia Math. 62 (1977), 92-101.

[26] R.H. Latter and A. Uchiyama, The atomic decomposition for parabolic $H^{p}$ spaces, Trans. Amer. Math. Soc. 253 (1979), 391-398.

[27] R.A. Macías and C. Segovia, A decomposition into atoms of distributions on spaces of homogeneous type, Adv. in Math. 33 (1979), 271-309.

[28] J. Malecki, G. Serafin, T. Zorawik, Fourier-Bessel heat kernel estimates. J. Math. Anal. Appl. 439 (2016), no. $1,91-102$

[29] A. Miyachi, $H^{p}$ space over open subsets of $R^{n}$, Studia Math., 95 (1990), 205-228.

[30] E.M. Ouhabaz, Analysis of heat equations on domains. London Mathematical Society Monographs Series, 31. Princeton University Press, Princeton, NJ, 2005.

[31] Z. Shen, $L^{p}$ estimates for Schrödinger operators with certain potentials, Ann. Inst. Fourier (Grenoble) 45 (1995), 513-546.

[32] L. Song and L. Yan, Maximal function characterizations for Hardy spaces associated to nonnegative selfadjoint operators on spaces of homogeneous type, J. Evol. Equ., to appear.

[33] L. Song and L. Yan, A maximal function characterization for Hardy spaces associated to nonnegative selfadjoint operators satisfying Gaussian estimates, Adv. Math. 287 (2016), 463-484.

[34] A. Uchiyama, A maximal function characterization of $H^{p}$ on the space of homogeneous type, Trans. Amer. Math. Soc. 262 (1980), no. 2, 579-592.

[35] D. Yang and Y. Zhou, Localized Hardy spaces $H^{1}$ related to admissible functions on RD-spaces and applications to Schrödinger operators, Trans. Amer. Math. Soc. 363 (2011), 1197-1239.

Department of Mathematics, Macquarie University, NSW 2109, Australia

E-mail address: the.bui@mq.edu.au, bt_anh80@yahoo.com

Department of Mathematics, Macquarie University, NSW 2109, Australia

E-mail address: xuan.duong@mq.edu.au

The School of Mathematics and Statistics, The Faculty of Science, The University of Sydney, NSW 2006, Australia

E-mail address: ken.1y@sydney.edu.au 